\title{
Recognition of Imidazoles by Strapped Zinc(II) Porphyrin Receptors: Insight into the Induced-Fit Mechanism
}

\author{
Jeremy Brandel, ${ }^{\dagger}$ Ali Trabolsi, $^{\dagger}$ Frédéric Melin, ${ }^{\ddagger}$ Mourad \\ Elhabiri, ${ }^{\dagger}$ Jean Weiss ${ }^{\star \ddagger}$ and Anne-Marie Albrecht-Gary* ${ }^{\dagger}$
}

$\dagger$ Laboratoire de Physico-Chimie Bioinorganique, ULP-CNRS (UMR 7177), Institut de Chimie, ECPM, 25, rue Becquerel, 67200 Strasbourg, France. Fax: 33 (0) 3902426 39; Tel: 33 (0) 390242638 .

\$ Laboratoire de Chimie des Ligands à Architecture Contrôlée, ULP-CNRS (UMR 7177) Institut de Chimie, 4, rue Blaise Pascal, 67000 Strasbourg, France. Fax: 33 (0) 3902414 31; Tel: 33 (0) 390241419.

\section{Supporting Information Available}

* To whom correspondence should be addressed: amalbre@chimie.u-strasbg.fr, jweiss@chimie.u-strasbg.fr

${ }^{\dagger}$ Laboratoire de Physico-Chimie Bioinorganique.

\$ Laboratoire de Chimie des Ligands à Architecture Contrôlée 
Starting Materials and Solvents. Zinc porphyrins $\left(\mathrm{ZnL}^{1}\right.$ and $\left.\mathrm{ZnTPP}\right)$ were synthesized according to a previously published procedure. ${ }^{1}$ Dichloroethane (Merck, 99,8 \% or CARLOERBA, 99,8 \% for spectroscopy) was freshly distillated over $\mathrm{CaH}_{2}$ prior to use. The imidazole substrates were used as purchased from commercial sources without further purification. All stock solutions were prepared using a AG 245 Mettler Toledo analytical balance (precision $0.01 \mathrm{mg}$ ) and the complete dissolution in dichloroethane was obtained with the help of ultrasonic bath. Their concentrations were calculated by weight. All these solutions were protected from daylight to avoid any photochemical degradation.

\section{$\mathbf{L}^{2}$ Ligand Synthesis.}

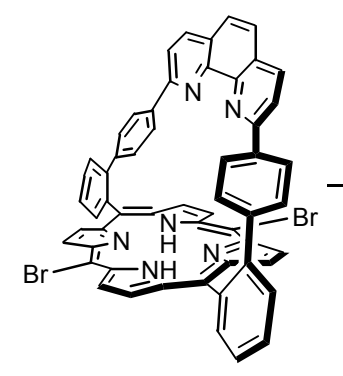

1

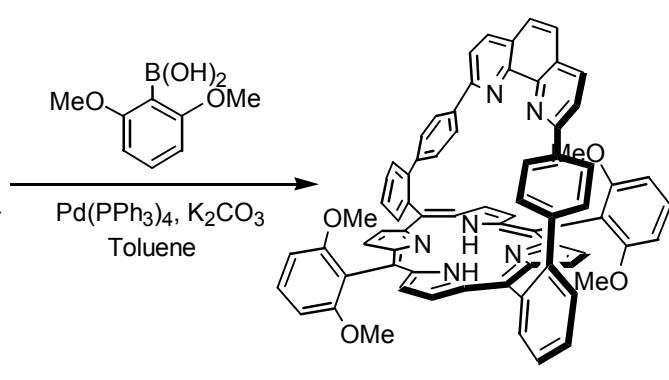

$\mathbf{L}^{2}$

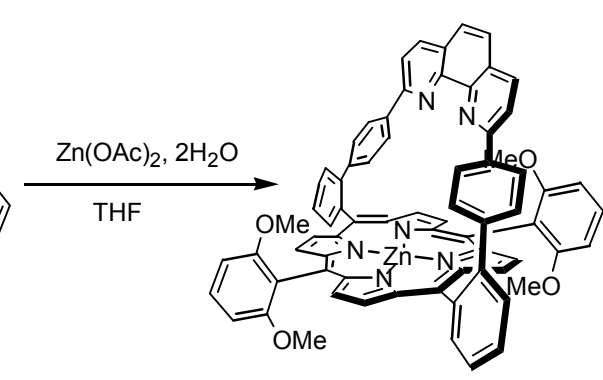

$\mathrm{ZnL}^{2}$

The starting dibromide compound $\mathbf{1}$ was obtained following an already published method. ${ }^{5}$

$\mathbf{L}^{2}$. To a degassed and dried mixture of the dibromide 1 (200 $\left.\mathrm{mg}, 0.2 \mathrm{mmol}\right)$, the $(2,6)$ dimethoxy-phenyl boronic acid (140 mg, $2.0 \mathrm{mmol})$, and $\mathrm{K}_{2} \mathrm{CO}_{3}(270 \mathrm{mg}, 2.0 \mathrm{mmol})$ in $20 \mathrm{ml}$ of toluene, were added, under argon, $30 \mathrm{mg}$ of $\mathrm{Pd}\left(\mathrm{PPh}_{3}\right)_{4}(0.02 \mathrm{mmol})$. After $20 \mathrm{~h}$ of reflux, the solvent was evaporated and the mixture was taken in $50 \mathrm{ml}$ of $\mathrm{CH}_{2} \mathrm{Cl}_{2}$. The solution was washed twice with brine and once with water, and dried over $\mathrm{Na}_{2} \mathrm{SO}_{4}$. Filtration and evaporation of the solvent yielded a red solid which was filtrated over $\mathrm{Al}_{2} \mathrm{O}_{3}$ (neutral, activity II-III, diameter $4 \mathrm{~cm}, \mathrm{~h}=10 \mathrm{~cm}$ ). Elution with $\mathrm{CH}_{2} \mathrm{Cl}_{2}$ yielded $123 \mathrm{mg}$ of the porphyrin $\mathbf{L}^{2}$ (0.12 mmol, 55\%). This compound was used in the following step without further purifications.

(1) Froidevaux, J.; Ochsenbein, P.; Bonin, M.; Schenk, K.; Maltese, P.; Gisselbrecht, J.P.; Weiss, J. J. Am. Chem. Soc. 1997, 119, 12362-12363. 
${ }^{1} \mathrm{H}$ NMR $\left(\mathrm{CDCl}_{3}, 300 \mathrm{MHz}\right): 8.65(\mathrm{~m}, 10 \mathrm{H}), 7.99$ (d, $\left.J=8.4 \mathrm{~Hz}, 2 \mathrm{H}\right), 7.85(\mathrm{~m}, 6 \mathrm{H}), 7.60$ (m, 4H), $7.52(\mathrm{~s}, 2 \mathrm{H}), 7.01(\mathrm{~m}, 2 \mathrm{H}), 6.93(\mathrm{~d}, J=8.3 \mathrm{~Hz}, 4 \mathrm{H}), 6.80(\mathrm{~d}, \mathrm{~J}=8.3 \mathrm{~Hz}, 2 \mathrm{H}), 6.66(\mathrm{~d}, J=$ $8.3 \mathrm{~Hz}, 4 \mathrm{H}), 3.67$ (s, 6H), $3.14(\mathrm{~s}, 6 \mathrm{H}),-2.10(\mathrm{~s}, 2 \mathrm{H})$.

$\mathbf{Z n L}^{2}$. A solution of $\mathbf{L}^{2}$ (123 mg, $\left.0.12 \mathrm{mmol}\right)$ and $\mathrm{Zn}(\mathrm{OAc})_{2}, 2 \mathrm{H}_{2} \mathrm{O}$ (250 mg, $\left.1.1 \mathrm{mmol}\right)$ in 20 $\mathrm{ml}$ of THF was refluxed for 3 hours under Argon. The solvent was removed and the crude product was dissolved in $\mathrm{CH}_{2} \mathrm{Cl}_{2}$. The solution was washed three times with water and dried over $\mathrm{Na}_{2} \mathrm{SO}_{4}$. The product was purified by chromatography over $\mathrm{Al}_{2} \mathrm{O}_{3}$ (neutral, activity II-III, diameter $4 \mathrm{~cm}, \mathrm{~h}=20 \mathrm{~cm})$. The product was eluted with $\mathrm{CH}_{2} \mathrm{Cl}_{2} /$ cyclohexane $(50 / 50)$ and recrystallized from $\mathrm{CH}_{2} \mathrm{Cl}_{2} / \mathrm{MeOH}$. Filtration yielded $100 \mathrm{mg}$ of a purple powder $(0.08 \mathrm{mmol}$, $70 \%)$.

${ }^{1} \mathrm{H} \mathrm{NMR}\left(\mathrm{CDCl}_{3}, 300 \mathrm{MHz}\right): 8.75(\mathrm{~d}, J=4.6 \mathrm{~Hz}, 4 \mathrm{H}), 8.72(\mathrm{~m}, 2 \mathrm{H}), 8.60(\mathrm{~d}, J=4.6 \mathrm{~Hz}, 4 \mathrm{H})$, 7.99 (d, $J=8.4 \mathrm{~Hz}, 2 \mathrm{H}), 7.85$ (m, 6H), 7.59 (m, 4H), 7.52 (s, 2H), 7.54 (s, 2H), 6.89 (m, 4H), $6.73(\mathrm{~d}, J=8.3 \mathrm{~Hz}, 4 \mathrm{H}), 6.51(\mathrm{~d}, J=8.3 \mathrm{~Hz}, 4 \mathrm{H}), 3.35$ (s, 6H), 3.30 (s, 6H).

C, $\mathrm{H}, \mathrm{N}$ analysis: \% calculated for $\mathrm{C}_{72} \mathrm{H}_{48} \mathrm{~N}_{6} \mathrm{O}_{4} \mathrm{Zn}+0.5 \mathrm{CH}_{2} \mathrm{Cl}_{2}+\mathrm{H}_{2} \mathrm{O}: \mathrm{C} 73.36, \mathrm{H} 4.33, \mathrm{~N}$ 7.08; found: C 73.48, H 3.91, N 6.84.

UV-Visible Titrations. The spectrophotometric titration of $\mathrm{ZnL}^{1}\left(\approx 2 \times 10^{-6} \mathrm{M}\right), \mathrm{ZnL}^{2}\left(\approx 10^{-6}\right.$ M) or ZnTPP $\left(\approx 10^{-6} \mathrm{M}\right)$ with $\mathbf{S n}(\mathrm{n}=1-5)$ were carried out in a Hellma quartz optical cell (1 or $2 \mathrm{~cm}$ ). Microvolumes of a concentrated solution of $\mathbf{S n}(\mathrm{n}=1-5)$ were added to $4 \mathrm{~mL}$ (for $l$ $=2 \mathrm{~cm}$ ) or $2 \mathrm{~mL}$ (for $l=1 \mathrm{~cm}$ ) of porphyrinic receptors with microliter Hamilton syringes (\#710 and \#750). Special care was taken to ensure that complete equilibration was attained. The corresponding UV-visible spectra were recorded from $290 \mathrm{~nm}$ to $700 \mathrm{~nm}$ on a Kontron Uvikon 941 spectrophotometer maintained at $25.0 \pm 0.2{ }^{\circ} \mathrm{C}$ by the flow of a Haake NB 22 thermostat.

Luminescence Titrations. Luminescence titrations were carried out on solutions of $\mathrm{Zn} \mathbf{L}^{1}$, $\mathrm{Zn \mathbf {L } ^ { 2 }}$ or $\mathrm{ZnTPP}\left(\approx 5 \times 10^{-7} \mathrm{M}\right)$ with an absorbance smaller than 0.1 at wavelengths $\geq \lambda_{\text {exc }}$ in order to avoid any errors due to the inner filter effect. The titration of $2 \mathrm{~mL}$ of the metalloporphyrins were carried out in a $1 \mathrm{~cm}$ Hellma quartz optical cell by addition of known microvolumes of solution of $\mathbf{S n}(n=1-5)$ with microliter Hamilton syringes (\#710 and \#750). The excitation wavelength corresponds to an isosbestic point (Soret band) between the free receptors and the pentacoordinated complexes. The zinc(II) porphyrin-centered luminescence 
spectra were recorded from $400 \mathrm{~nm}$ to $800 \mathrm{~nm}$ on a Perkin-Elmer LS-50B maintained at 25.0 $\pm 0.2{ }^{\circ} \mathrm{C}$ by the flow of a Haake FJ thermostat. The slit width was set at $15 \mathrm{~nm}$ for both the excitation and the emission.

Processing of the spectrophotometric data. The spectrophotometric data were processed with both the Letagrop-Spefo and Specfit programs, which adjust the stability constants and the corresponding extinction coefficients of the species formed at equilibrium. LetagropSpefo $^{2-4}$ uses the Newton-Raphson algorithm to solve mass balance equations and a pitmapping method to minimize the errors and determine the best values of the parameters. Specfit ${ }^{5-8}$ uses factor analysis to reduce the absorbance matrix and to extract the eigenvalues prior to the multiwavelength fit of the reduced data set according to the Marquardt algorithm. 9,10

Kinetics of formation. All kinetic measurements were performed in 1,2-dichloroethane using a stopped-flow spectrophotometer (Applied Photophysic SX-18MV). The reactants were thermostated at $25.0 \pm 0.2{ }^{\circ} \mathrm{C}$ (Lauda M12 thermostat) and mixed in a $1 \mathrm{~cm}$ optical cell. For fluorescence measurements, the excitation monochromator slits were set at $3 \mathrm{~nm}$, and the excitation wavelength corresponds to an isosbestic point (Soret or Q bands) between the free receptors and the pentacoordinated complexes. A $550 \mathrm{~nm}$ "cut off" filter (transparent to light with a wavelength higher than $550 \mathrm{~nm}$ ) was used for the emitted light. For absorption, the chosen wavelength corresponds to the largest amplitude. The data sets, averaged out of at least three replicates, were recorded and analyzed with the commercial software Biokine. ${ }^{11}$ This program fits up to three exponential functions to the experimental curves with the

(2) Sillen, L.G. Acta Chem. Scand. 1964, 18, 1085-1098.

(3) Sillen, L.G.; Warnqvist, B. Ark. Kemi. 1968, 31, 377-390.

(4) Havel, J. Pure Appl. Chem. 1972, 34, 370-388.

(5) Gampp, H.; Maeder, M.; Meyer, C.J.; Zuberbühler, A.D. Talanta 1985, 32, 95-101.

(6) Rossoti, F.J.C.; Rossoti, H.S.; Whewell, R.J. J. Inorg. Nucl. Chem. 1971, 33, 2051-2065.

(7) Gampp, H.; Maeder, M.; Meyer, C.J.; Zuberbühler, A.D. Talanta 1985, 32, 257-264.

(8) Gampp, H.; Maeder, M.; Meyer, C.J.; Zuberbühler, A.D. Talanta 1986, 33, 943-951.

(9) Marquardt, D.W. J. Soc. Indust. Appl. Math. 1963, 11, 431-441.

(10) Maeder, M.; Zuberbühler, A.D. Anal. Chem. 1990, 62, 2220-2224.

(11) Bio-Logic Company, Ed. Bio-Logic Company, Echirolles, 1991. 
Simplex algorithm ${ }^{12}$ after initialization with the Padé-Laplace method. ${ }^{13}$ The activation parameters for formation and dissociation paths were derived from conventional Eyring plots of kinetic data at different temperatures (in the range of $22-37^{\circ} \mathrm{C}$ ). Variations of the pseudofirst order rate constants with the analytical concentrations of reagents were fitted using commercial programs (Origin 5.0 $0^{14}$ ).

(12) Nelder, J.A.; Mead, R. The Computer Journal 1965, 7, 308-313.

(13) Yeramian, E.; Claverie, P. Nature 1987, 326, 169-174.

(14) Microcal $^{\mathrm{TM}}$ Origin ${ }^{\mathrm{TM}}$, Microcal Software, Inc., Northampton, USA. 


\begin{tabular}{lcll}
\hline \multicolumn{1}{c}{ Receptors } & \multicolumn{2}{c}{$\mathrm{ZnL}^{1}$} & \multicolumn{2}{c}{$\mathrm{ZnTPP}$} \\
Substrates & \multicolumn{2}{c}{$\log K_{\mathrm{ZnL}{ }^{{ }}-\mathrm{s}}$} & $\log K_{\text {ZnTPP-S }}$ \\
\hline S1 & $4.3(3)$ & $3.6(1)$ & $4.7(1)$ \\
S2 & $5.9(1)$ & $7.0(2)$ & $4.47(6)$ \\
S3 & $7.6(4)$ & $7.7(5)$ & $4.9(1)$ \\
S4 & $3.92(7)$ & $2.3(3)$ & $2.54(6)$ \\
S5 & $5.3(4)$ & $5.14(4)$ & $3.8(2)$
\end{tabular}

Table S1. Averaged Stability Constants $\left(\log K_{Z n \boldsymbol{L}^{n}-S}\right)$ of $Z n \boldsymbol{L}^{n}-\boldsymbol{S}$ and $\left(\log K_{Z n T P P-S}\right)$ of ZnTPP$\boldsymbol{S}$ Complexes: Solvent: 1,2-dichloroethane; $T=25.0(2){ }^{\circ} \mathrm{C}$. Stability constants determined from UV-vis. absorption and fluorescence spectrophotometric titrations. Uncertainties $=3 \sigma$.

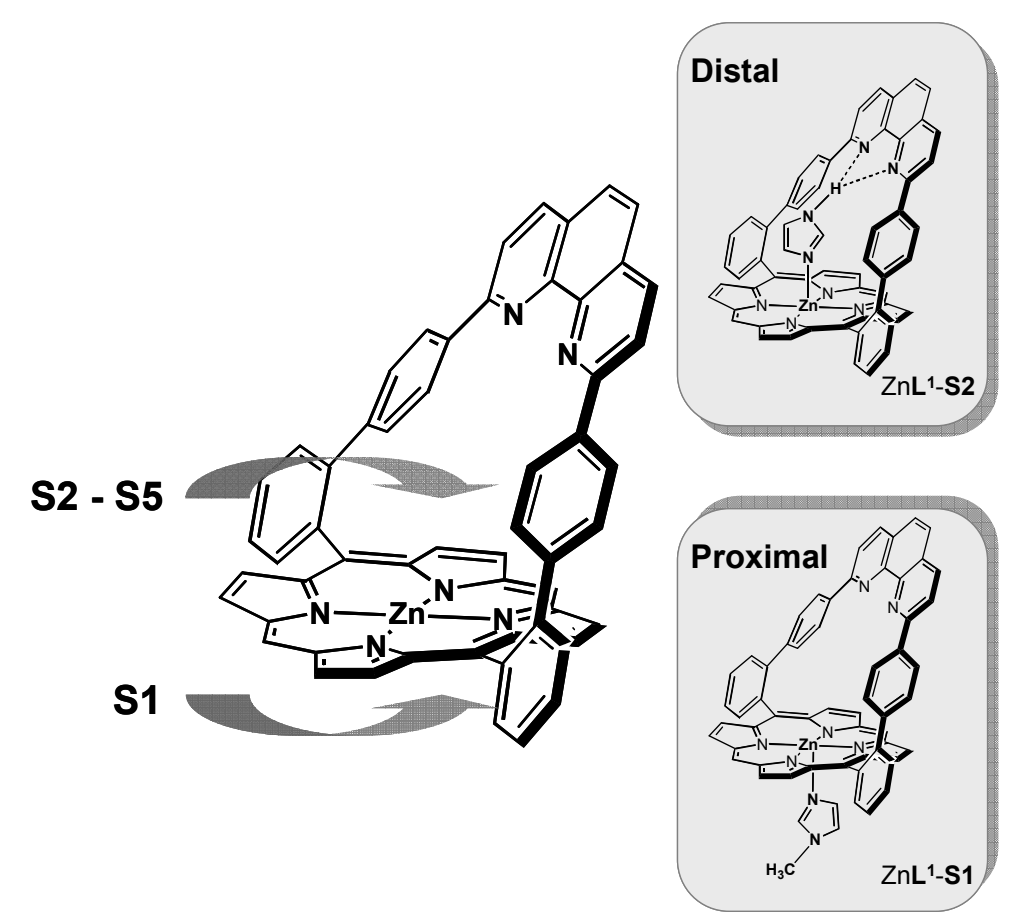

Proximal/distal recognition of imidazole substrates $\boldsymbol{S 1 - S 5}$ with receptors $Z n \boldsymbol{L}^{1}$ and $Z n \boldsymbol{L}^{2}$. 


\section{Spectrophotometric Titrations (UV-vis Absorption) of $\mathrm{ZnL}^{1}$ Receptor by Substrates $\mathbf{S}$.}

\begin{tabular}{lll}
\hline & {$[\mathrm{S}] /(\mathrm{M})$} & {$\left[\mathrm{ZnL}^{1}\right] /(\mathrm{M})$} \\
\hline & $9.50 \times 10^{-3}$ & $2.06 \times 10^{-6}$ \\
& & \\
& & \\
& & \\
& & \\
\end{tabular}

Table S2. Experimental conditions for the UV-visible absorption spectrophotometric titrations of $Z_{n} \boldsymbol{L}^{1}$ receptor by substrates $\boldsymbol{S 1 - S 5}$. Solvent: 1,2-dichloroethane; $T$ $=25.0(2){ }^{\circ} \mathrm{C}$.

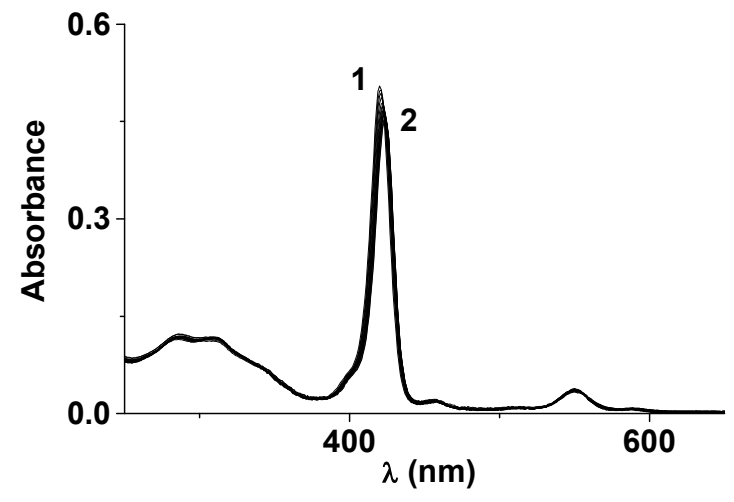

a)

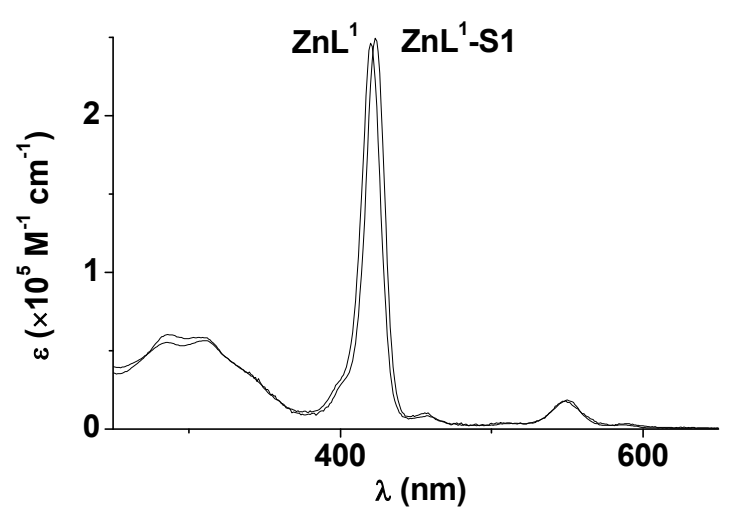

b)

Figure S1. a) UV-visible absorption spectrophotometric titration of $\mathrm{ZnL}^{1}$ receptor versus 1-methyl-1H-imidazole concentrations (noted S1). Solvent: 1,2dichloroethane; $T=25.0(2){ }^{\circ} \mathrm{C} ; \mathrm{l}=1 \mathrm{~cm}$; (1) $\left[\mathrm{Zn} \boldsymbol{L}^{1}\right]_{\text {tot }}=2.06 \times 10^{-6} \mathrm{M}$; (2) $[\mathbf{S 1}]_{\text {tot }} /[\mathrm{ZnL}]_{\text {tot }}=46422$.

b) Electronic spectra. 


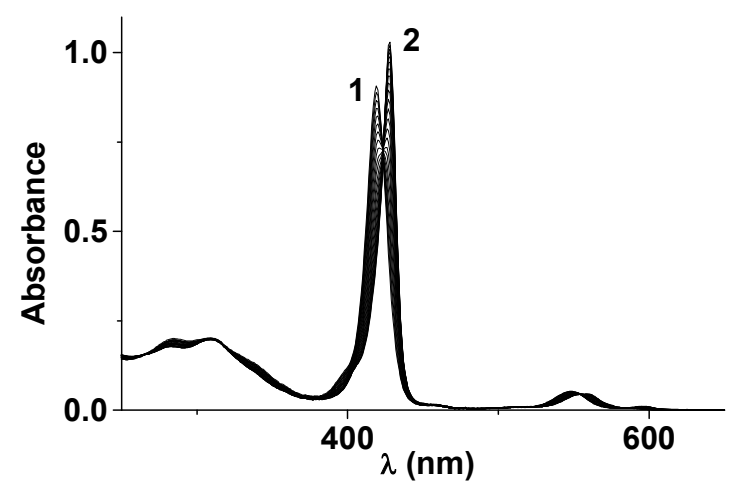

a)

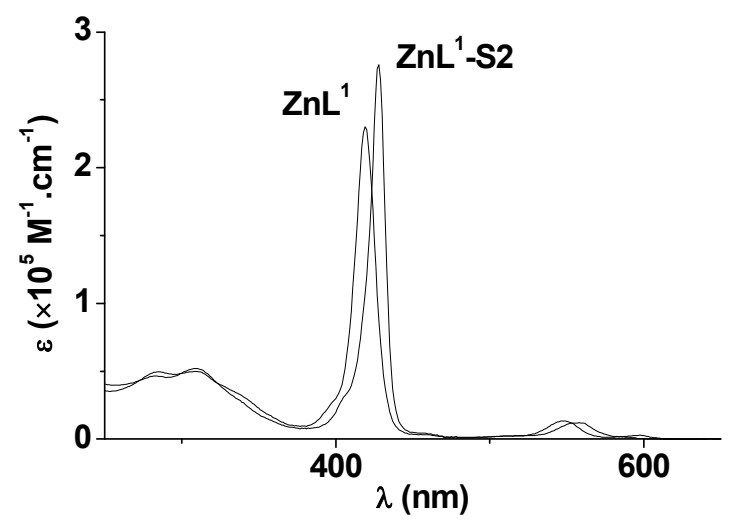

b)

Figure S2. a) UV-visible absorption spectrophotometric titration of $\mathrm{ZnL}^{1}$ receptor versus 1H-imidazole concentrations (noted S2). Solvent: 1,2-dichloroethane; $T=$ $25.0(2){ }^{\circ} \mathrm{C} ; \mathrm{l}=2 \mathrm{~cm}$; (1) $\left[\mathrm{Zn} \boldsymbol{L}^{1}\right]_{t o t}=2.01 \times 10^{-6} \mathrm{M}$; (2) $[\boldsymbol{S} 2]_{t o t} /\left[\mathrm{Zn} \boldsymbol{L}^{1}\right]_{t o t}=$ 1.26 .

b) Electronic spectra.

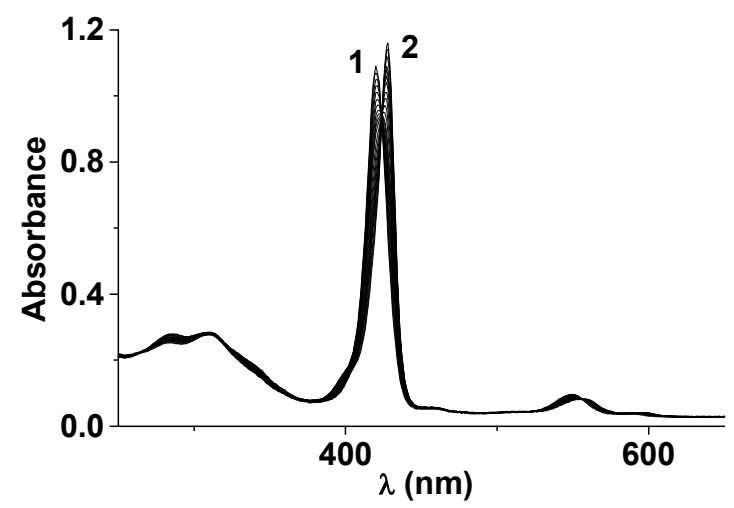

a)

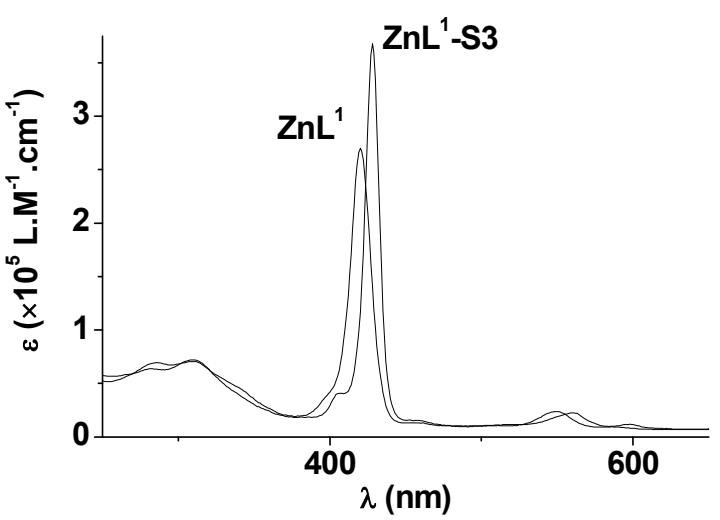

b)

Figure S3. a) UV-visible absorption spectrophotometric titration of $\mathrm{ZnL}^{1}$ receptor versus 2-methyl-1H-imidazole concentrations (noted S3). Solvent: 1,2dichloroethane; $T=25.0(2){ }^{\circ} \mathrm{C} ; \mathrm{l}=2 \mathrm{~cm}$; (1) $\left[\mathrm{Zn} \boldsymbol{L}^{1}\right]_{\text {tot }}=2.01 \times 10^{-6} \mathrm{M}$; (2) $[\boldsymbol{S} 3]_{\text {tot }} /\left[\mathrm{Zn} \boldsymbol{L}^{1}\right]_{\text {tot }}=2.8$.

b) Electronic spectra. 


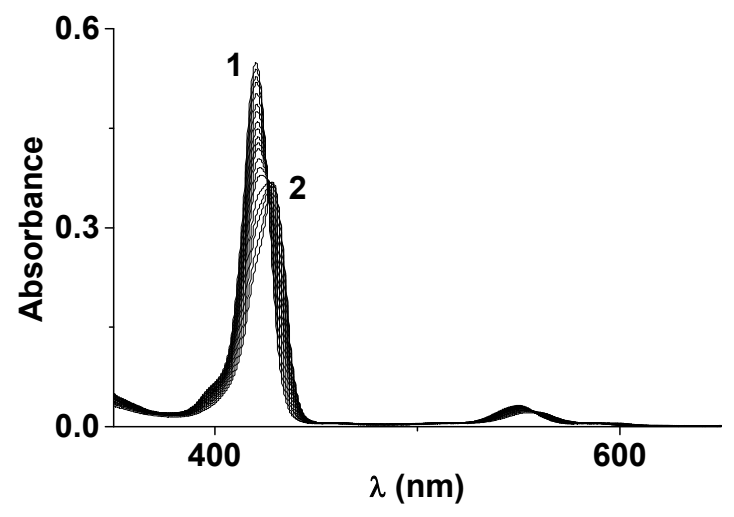

a)

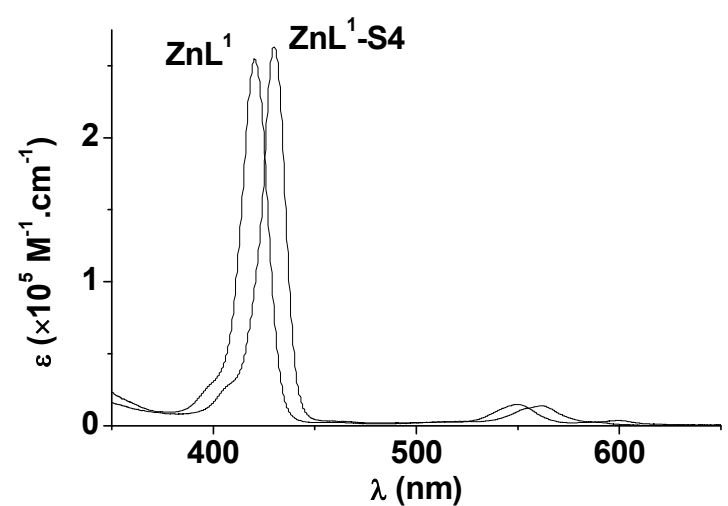

b)

Figure S4. a) UV-visible absorption spectrophotometric titration of $\mathrm{ZnL}^{1}$ receptor versus 2-phenyl-1H-imidazole concentrations (noted S4). Solvent: 1,2dichloroethane; $T=25.0(2){ }^{\circ} \mathrm{C} ; \mathrm{l}=1 \mathrm{~cm}$; (1) $\left[\mathrm{Zn} \boldsymbol{L}^{1}\right]_{\text {tot }}=2.16 \times 10^{-6} \mathrm{M}$; (2) $[\boldsymbol{S} 4]_{\text {tot }} /\left[\mathrm{Zn} \boldsymbol{L}^{1}\right]_{\text {tot }}=175$.

b) Electronic spectra.

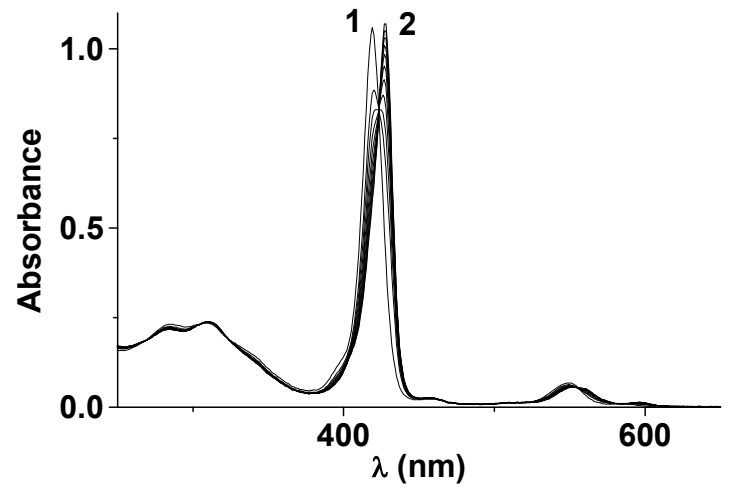

a)

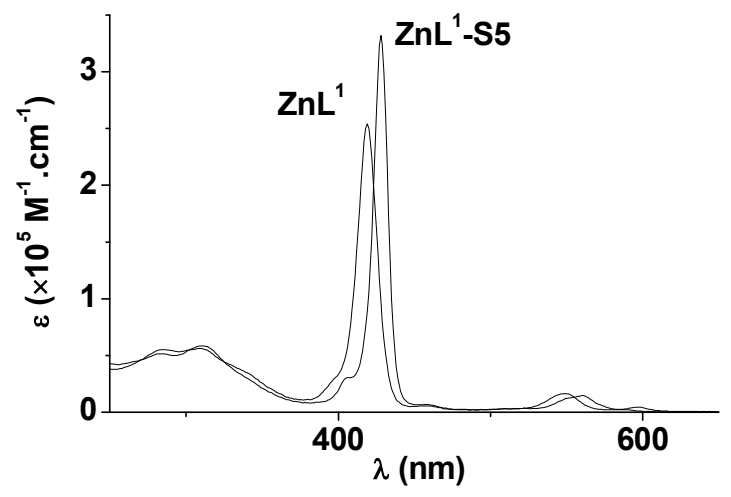

b)

Figure S5. a) UV-visible absorption spectrophotometric titration of $Z n \boldsymbol{L}^{1}$ receptor versus $N$-acetyl-(L)-histidine methyl ester concentrations (noted S5). Solvent: 1,2dichloroethane; $T=25.0(2){ }^{\circ} \mathrm{C} ; \mathrm{l}=2 \mathrm{~cm}$; (1) $\left[\mathrm{Zn} \boldsymbol{L}^{1}\right]_{\text {tot }}=2.06 \times 10^{-6} \mathrm{M}$; (2) $[\mathbf{S 5}]_{\text {tot }} /[\mathbf{Z n L}]_{\text {tot }}=9.34$.

b) Electronic spectra. 


\section{Luminescence Titrations of $\mathrm{ZnL}^{1}$ Receptor by Substrates S.}

\begin{tabular}{|c|c|c|c|}
\hline Substrates & {$[\mathbf{S}] / \mathrm{M}$} & {$\left[\mathbf{Z n L}^{1}\right] / \mathrm{M}$} & $\lambda_{\mathrm{exc}} / \mathrm{nm}$ \\
\hline S1 & $4.01 \times 10^{-3}$ & $5.22 \times 10^{-7}$ & 422 \\
\hline S2 & $3.83 \times 10^{-5}$ & $6.50 \times 10^{-7}$ & 424 \\
\hline S3 『 & $1.18 \times 10^{-5}$ & $5.22 \times 10^{-7}$ & 423 \\
\hline S4 & $7.53 \times 10^{-3}$ & $5.42 \times 10^{-7}$ & 425 \\
\hline S5 & $2.85 \times 10^{-4}$ & $5.22 \times 10^{-7}$ & 423 \\
\hline
\end{tabular}

Table S3. Experimental conditions for luminescence titrations of $\mathrm{Zn \boldsymbol {L } ^ { 1 }}$ receptor by substrates S1-S5. Solvent : 1,2-dichloroethane; $T=25.0(2){ }^{\circ} \mathrm{C}$; the slit width was set at $15 \mathrm{~nm}$ for both the excitation and the emission.

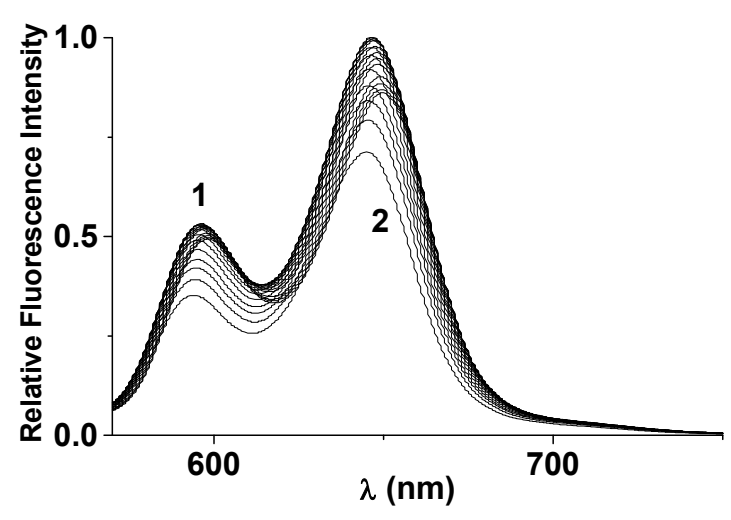

a)

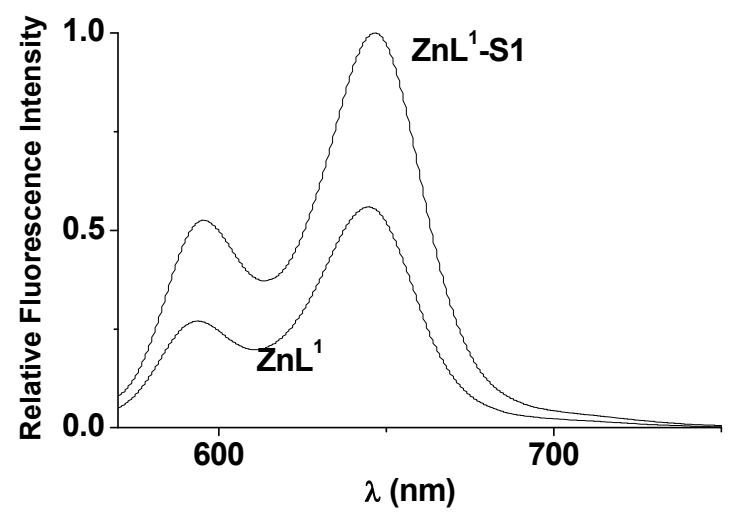

b)

Figure S6. a) Spectrofluorimetric titration of $Z n \boldsymbol{L}^{1}$ receptor versus 1-methyl-1H-imidazole concentrations (noted S1). Solvent: 1,2-dichloroethane; $T=25.0(2){ }^{\circ} \mathrm{C} ; \lambda_{\text {exc }}=$ $422 \mathrm{~nm}$; (1) $\left[\mathrm{Zn} \boldsymbol{L}^{1}\right]_{\text {tot }}=5.22 \times 10^{-7} \mathrm{M}$; (2) $[\boldsymbol{S} 1]_{\text {tot }} /\left[\mathrm{Zn} \boldsymbol{L}^{1}\right]_{\text {tot }}=864.2$.

b) Relative recalculated luminescence spectra. 


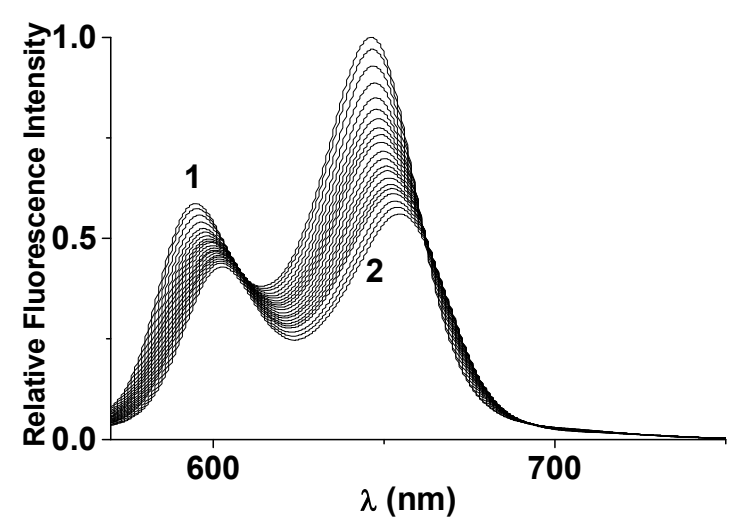

a)

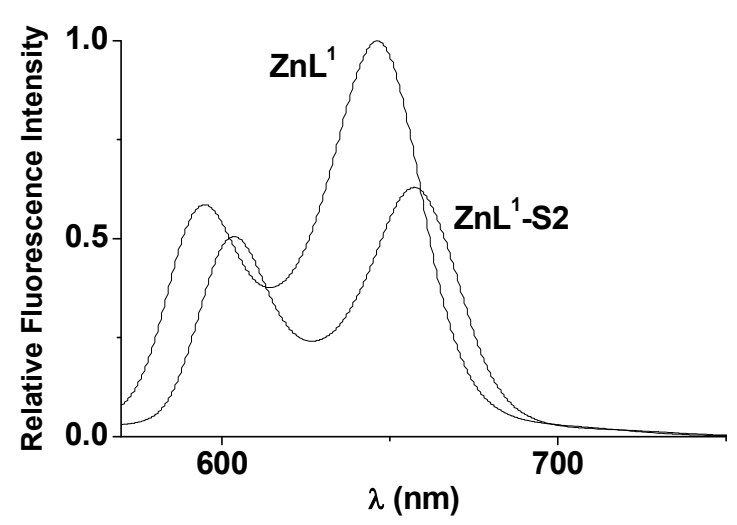

b)

Figure S7. a) Spectrofluorimetric titration of $\mathrm{ZnL}^{1}$ receptor versus 1 H-imidazole concentrations (noted $\boldsymbol{S} 2$ ). Solvent: 1,2-dichloroethane; $T=25.0(2){ }^{\circ} \mathrm{C} ; \lambda_{\text {exc }}=$ $424 \mathrm{~nm}$; (1) $\left[\mathrm{Zn} \boldsymbol{L}^{1}\right]_{\text {tot }}=6.50 \times 10^{-7} \mathrm{M}$; (2) $[\boldsymbol{S} 2]_{\text {tot }} /\left[\mathrm{Zn} \boldsymbol{L}^{1}\right]_{\text {tot }}=11.0$.

b) Relative recalculated luminescence spectra.

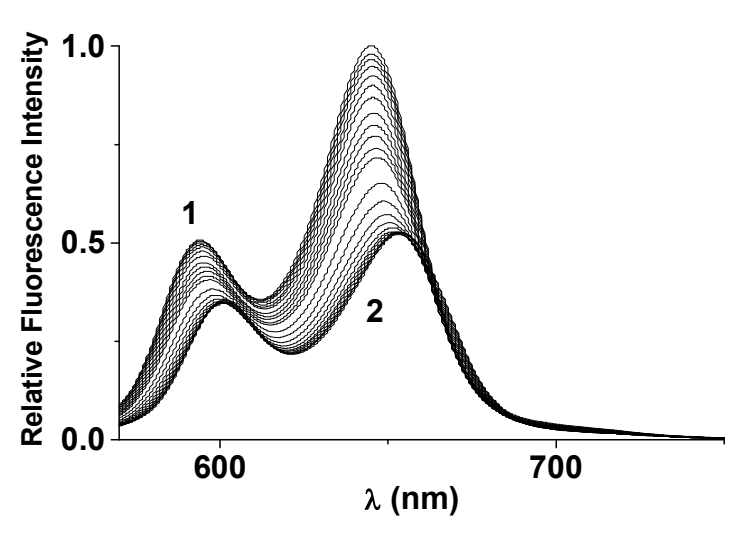

a)

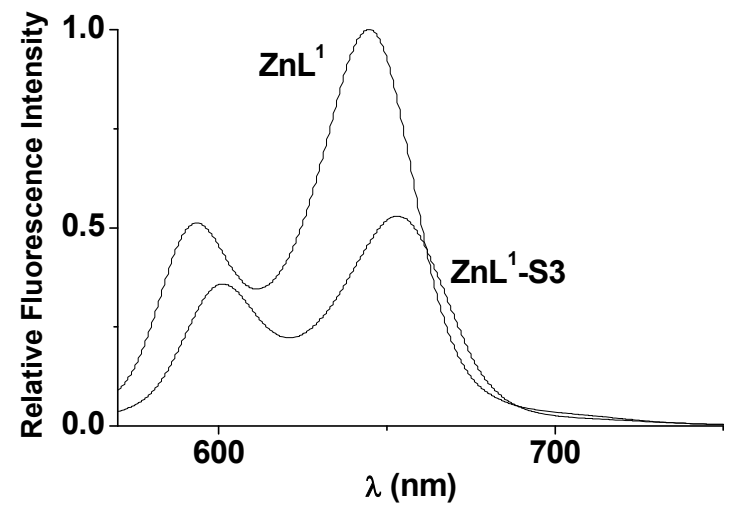

b)

Figure S8. a) Spectrofluorimetric titration of $Z n \boldsymbol{L}^{1}$ receptor versus 2-methyl-1H-imidazole concentrations (noted S3). Solvent: 1,2-dichloroethane; $T=25.0(2){ }^{\circ} \mathrm{C} ; \lambda_{\text {exc }}=$ $423 \mathrm{~nm}$; (1) $\left[\mathrm{Zn} \boldsymbol{L}^{1}\right]_{\text {tot }}=5.22 \times 10^{-7} \mathrm{M}$; (2) $[\mathbf{S} 3]_{\text {tot }} /\left[\mathrm{Zn} \boldsymbol{L}^{1}\right]_{\text {tot }}=2.7$.

b) Relative recalculated luminescence spectra. 


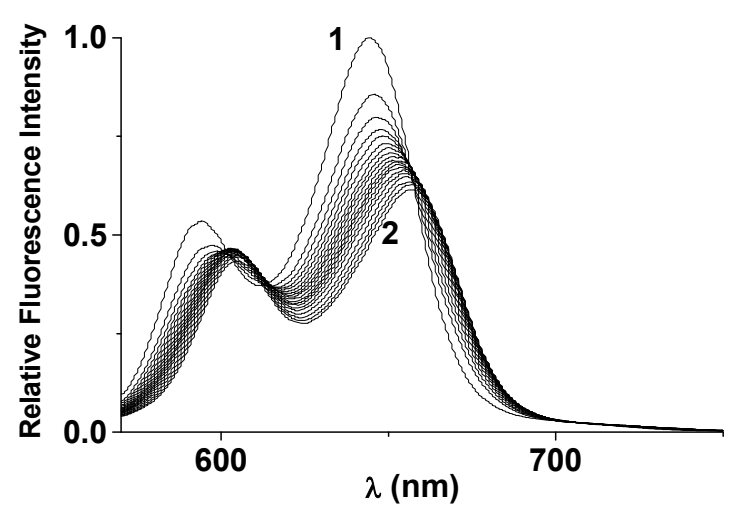

a)

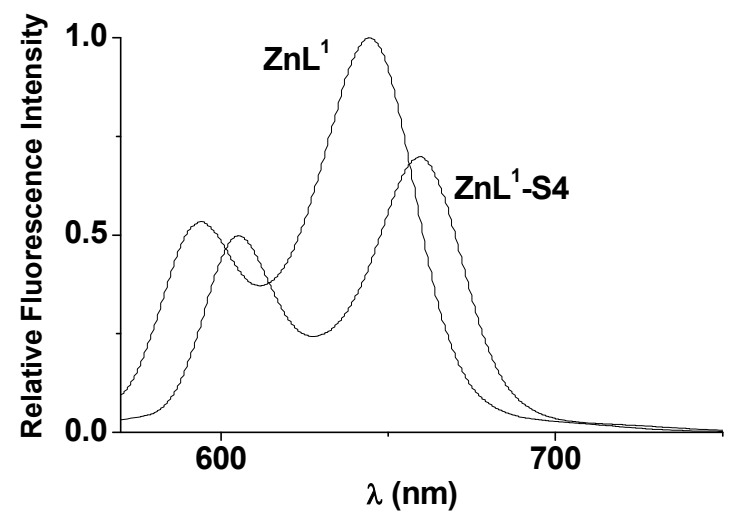

b)

Figure S9. a) Spectrofluorimetric titration of $Z n \mathbf{L}^{1}$ receptor versus 2-phenyl-1H-imidazole concentrations (noted $\boldsymbol{S 4}$ ). Solvent: 1,2-dichloroethane; $T=25.0(2){ }^{\circ} \mathrm{C} ; \lambda_{\text {exc }}=$ $425 \mathrm{~nm}$; (1) $\left[\mathrm{Zn \boldsymbol {L } ^ { 1 }}\right]_{\text {tot }}=5.42 \times 10^{-7} \mathrm{M}$; (2) $[\mathbf{S} 4]_{\text {tot }} /\left[\mathrm{Zn \boldsymbol {L } ^ { 1 }}\right]_{\text {tot }}=1062.8$.

b) Relative recalculated luminescence spectra.

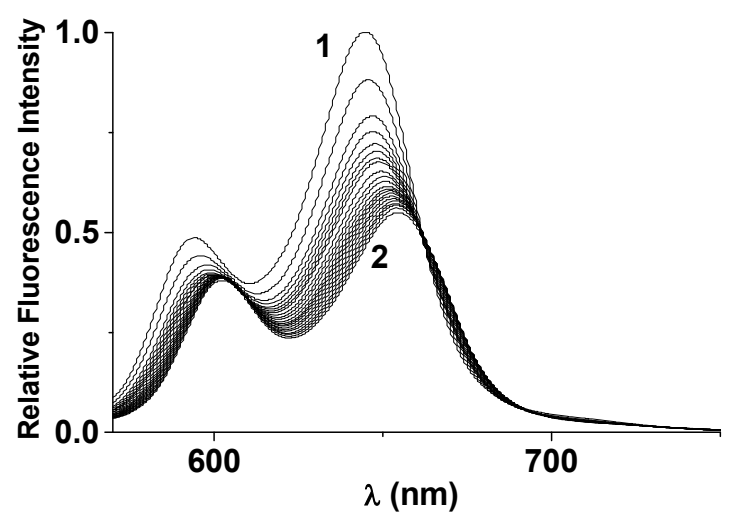

a)

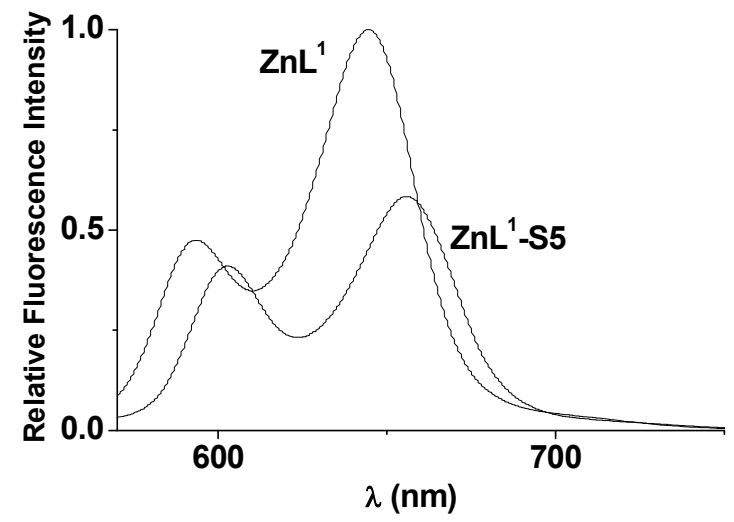

b)

Figure S10. a) Spectrofluorimetric titration of $Z n L^{1}$ receptor versus $N$-acetyl-(L)-histidine methyl ester concentrations (noted S5). Solvent: 1,2-dichloroethane; $T=$ $25.0(2){ }^{\circ} \mathrm{C} ; \lambda_{\text {exc }}=423 \mathrm{~nm}$; (1) $\left[\mathrm{Zn} \boldsymbol{L}^{1}\right]_{\text {tot }}=5.22 \times 10^{-7} \mathrm{M}$; (2) $[\mathbf{S 5}]_{\text {tot }} /\left[\mathrm{Zn} \boldsymbol{L}^{1}\right]_{\text {tot }}$ $=65.4$.

b) Relative recalculated luminescence spectra. 


\section{Kinetic Studies (Receptor $\mathrm{ZnL}^{1}$ and Substrate S3)}

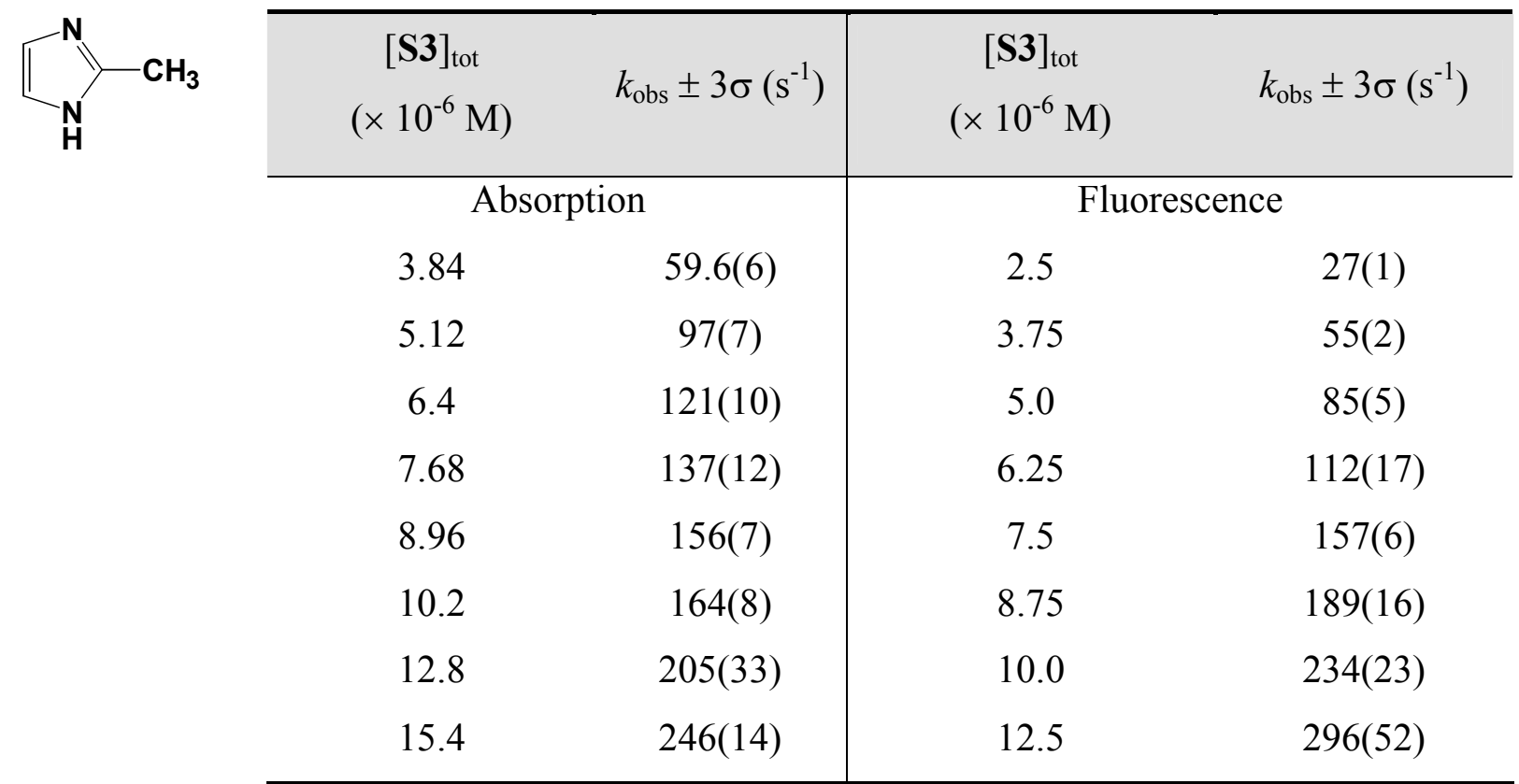

Table S4. Variation of the pseudo-first order rate constants versus $[\mathbf{S} 3]_{\text {tot }}$ for $\mathrm{ZnL} \boldsymbol{L}^{\mathbf{I}}-\mathbf{S} \mathbf{3}$ complex. Solvent: 1,2-dichloroethane; $T=25.0(2){ }^{\circ} \mathrm{C}$; a) Absorption: $\left[\mathrm{ZnL}^{1}\right]$ $=2.66 \times 10^{-7} \mathrm{M} ; \lambda=428 \mathrm{~nm} ;$ b) Fluorescence: $\left[\mathrm{Zn} \boldsymbol{L}^{1}\right]=2.51 \times 10^{-7} \mathrm{M}, \lambda_{\text {exc }}=$ $423 \mathrm{~nm}$.

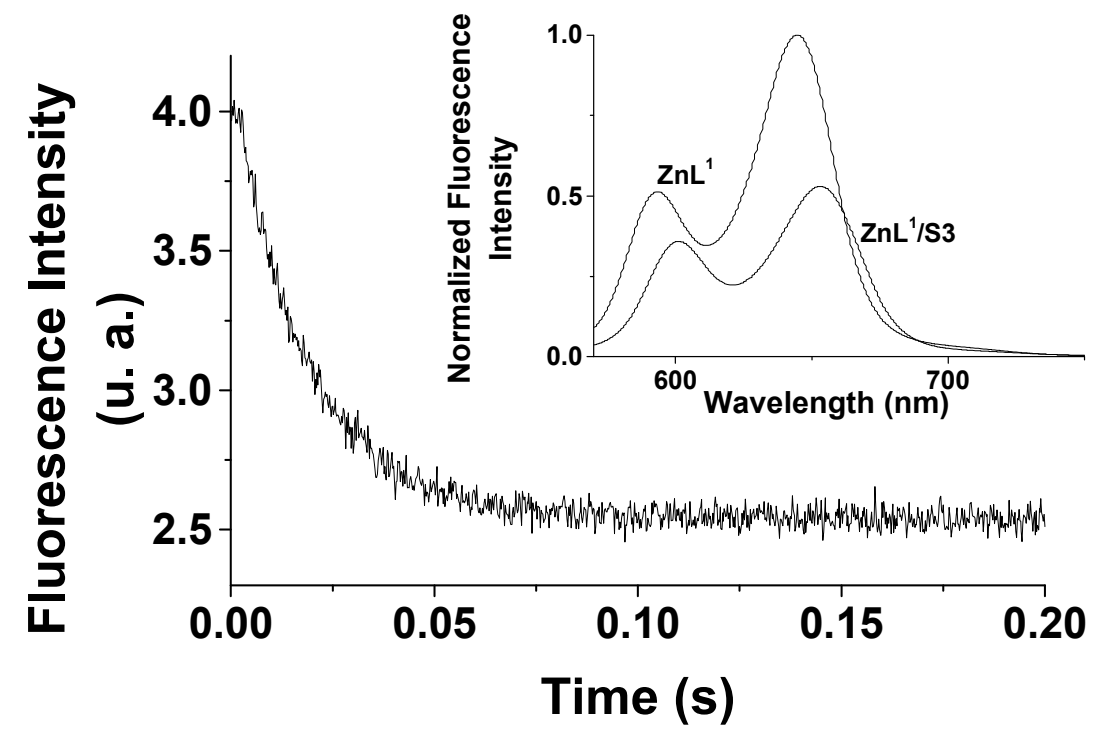

Figure S11. Kinetic trace recorded for the formation of complex $Z n \boldsymbol{L}^{1}-\mathbf{S} 3$ and measured by luminescence spectrophotometry. Solvent: 1,2-dichloroethane; $T=25.0(2){ }^{\circ} \mathrm{C}$; $\left[Z n \boldsymbol{L}^{1}\right]_{\text {tot }}=2.0 \times 10^{-7} \mathrm{M} ;[\mathbf{S} 3]_{\text {tot }}=2.0 \times 10^{-6} \mathrm{M} ; \lambda_{\text {exc }}=423 \mathrm{~nm}$. Inset: Normalized recalculated luminescence spectra of $Z n L^{1}$ and $Z n L^{1}-S 3$ species; $\lambda_{\text {exc }}=423 \mathrm{~nm}$. 


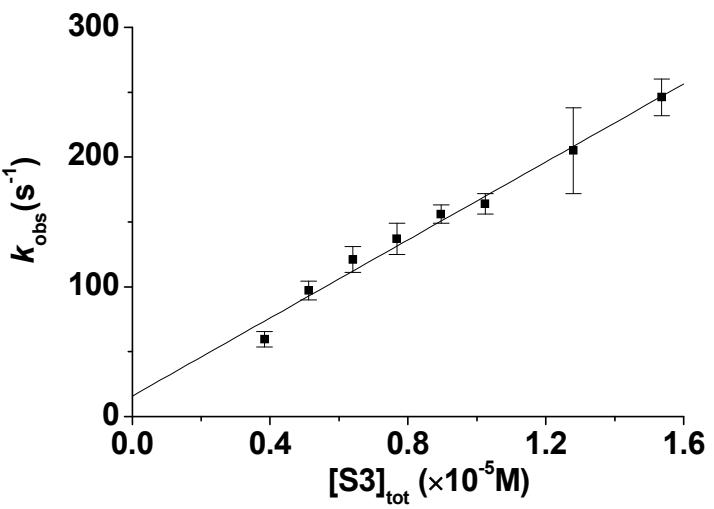

a)

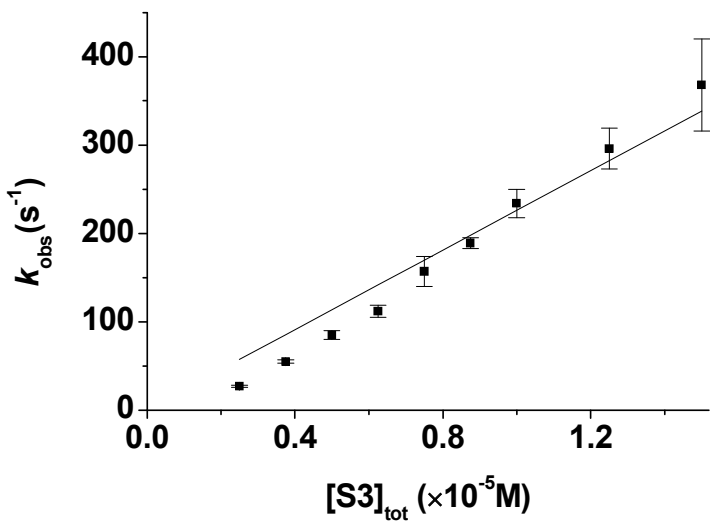

b)

Figure S12. Variation of the pseudo-first order rate constants $\left(k_{o b s}\right)$ for the formation of $\mathrm{ZnL} \boldsymbol{L}^{1}$-S3 complex versus [S3] ${ }_{\text {tot }}$. Solvent: 1,2-dichloroethane; $T=25.0(2){ }^{\circ} \mathrm{C}$; a) absorption: $\left[\mathrm{ZnL}^{1}\right]=2.66 \times 10^{-7} \mathrm{M} ; \lambda=428 \mathrm{~nm}$; b) Fluorescence: $\left[\mathrm{Zn} \boldsymbol{L}^{1}\right]$ $=2.51 \times 10^{-7} \mathrm{M} ; \lambda_{\text {exc }}=423 \mathrm{~nm}$. 


\section{Kinetic Studies (Receptor $\mathrm{ZnL}^{1}$ and Substrate S4)}

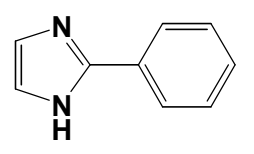

\begin{tabular}{cc|cc}
\hline $\begin{array}{c}{[\mathbf{S 4}]_{\text {tot }}} \\
\left(\times 10^{-4} \mathrm{M}\right)\end{array}$ & $k_{\text {obs }} \pm 3 \sigma\left(\mathrm{s}^{-1}\right)$ & $\begin{array}{c}{[\mathbf{S 4}]_{\text {tot }}} \\
\left(\times 10^{-5} \mathrm{M}\right)\end{array}$ & $k_{\text {obs }} \pm 3 \sigma\left(\mathrm{s}^{-1}\right)$ \\
\hline \multicolumn{2}{c|}{ Absorption } & \multicolumn{2}{|c}{ Fluorescence } \\
0.38 & $1.96(3)$ & 0.50 & $1.39(8)$ \\
0.51 & $2.18(9)$ & 1.0 & $1.52(8)$ \\
0.76 & $2.7(3)$ & 1.50 & $1.67(3)$ \\
1.01 & $3.3(2)$ & 2.50 & $1.88(3)$ \\
1.52 & $4.1(2)$ & 5.0 & $2.51(8)$ \\
2.03 & $4.44(7)$ & 10.0 & $3.92(8)$ \\
2.53 & $5.08(6)$ & 15.0 & $5.04(8)$ \\
\hline
\end{tabular}

Table S5. Variation of the pseudo-first order rate constants versus $[\mathbf{S 4}]_{\text {tot }}$ for $\mathrm{ZnL} \boldsymbol{L}^{\mathbf{I}} \mathbf{- S 4}$ complex. Solvent: 1,2-dichloroethane; $T=25.0(2){ }^{\circ} \mathrm{C}$; a) Absorption: $\left[\mathrm{Zn} \boldsymbol{L}^{1}\right]$ $=5.23 \times 10^{-7} \mathrm{M} ; \lambda=432 \mathrm{~nm}$; b) Fluorescence: $\left[\mathrm{ZnL}^{1}\right]=4.98 \times 10^{-7} \mathrm{M} ; \lambda_{\text {exc }}=$ $425 \mathrm{~nm}$.

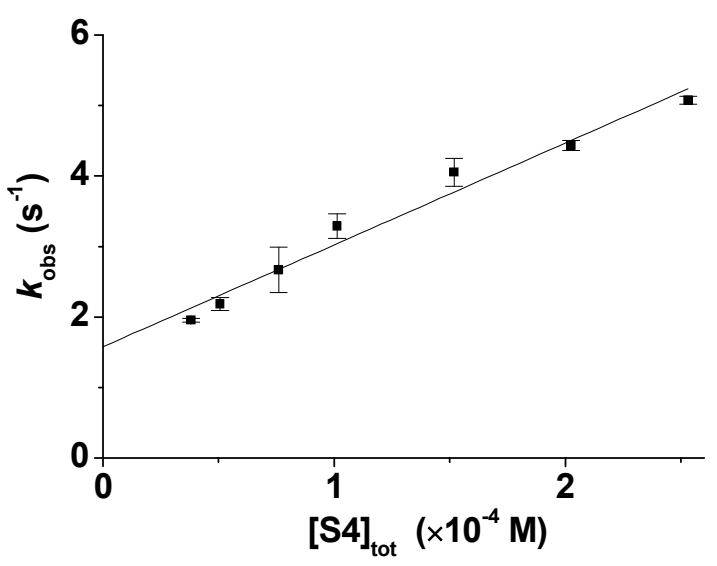

a)

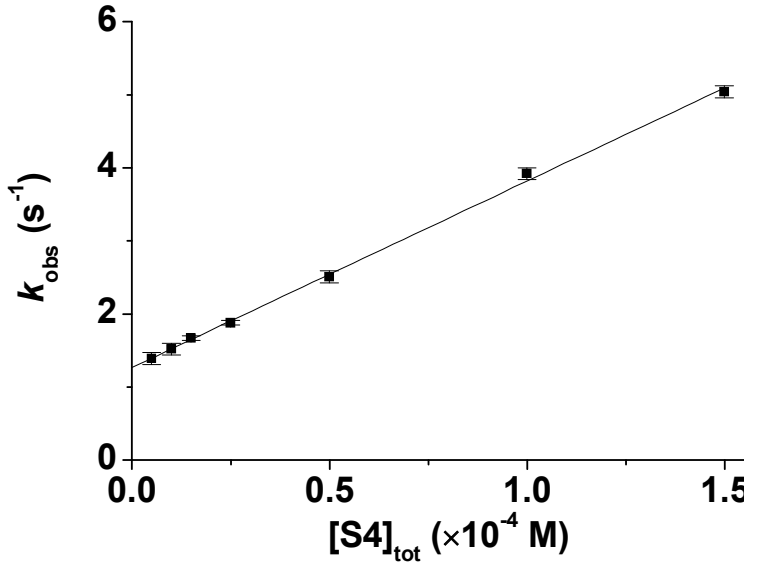

b)

Figure S13. Variation of the pseudo-first order rate constants $\left(k_{o b s}\right)$ for the formation of $\mathrm{Zn} \boldsymbol{L}^{\boldsymbol{1}}$-S4 complex versus $[\boldsymbol{S 4}]_{\text {tot }}$. Solvent: 1,2-dichloroethane; $T=25.0(2){ }^{\circ} \mathrm{C}$; a) absorption: $\left[\mathrm{Zn} \boldsymbol{L}^{1}\right]=5.23 \times 10^{-7} \mathrm{M} ; \lambda=432 \mathrm{~nm}$; b) fluorescence: $\left[\mathrm{Zn} \boldsymbol{L}^{1}\right]=$ $4.98 \times 10^{-7} \mathrm{M} ; \lambda_{\text {exc }}=425 \mathrm{~nm}$. 


\section{Kinetic Studies (Receptor $\mathrm{ZnL}^{1}$ and Substrate S5)}

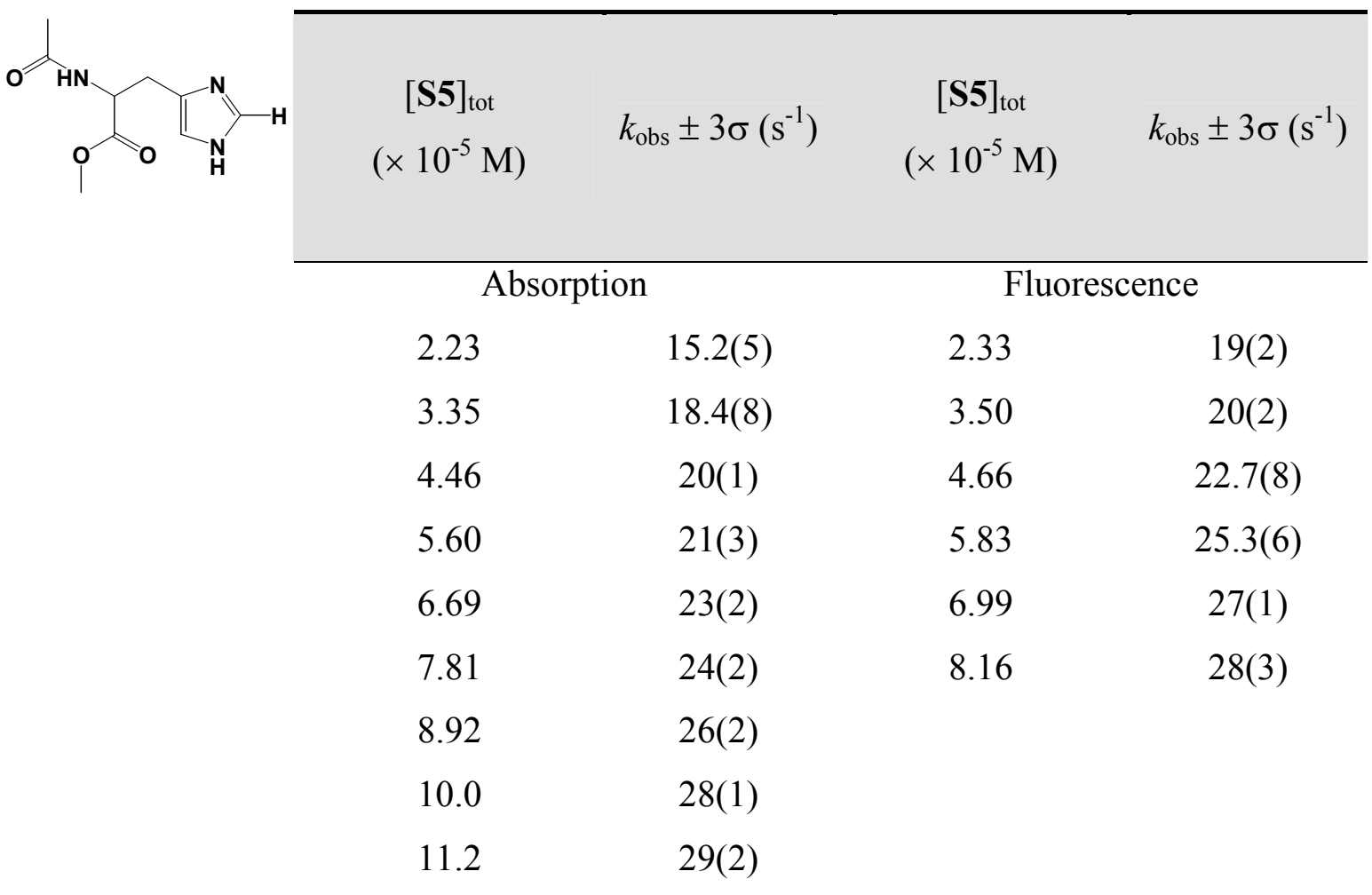

Table S6. Variation of the pseudo-first order rate constants versus $[\mathbf{S 5}]_{\text {tot }}$ for $\mathrm{ZnL} \boldsymbol{L}_{-\mathbf{S}} \mathbf{5}$ complex. Solvent: 1,2-dichloroethane; $T=25.0(2){ }^{\circ} \mathrm{C}$; a) Absorption: $\left[\mathrm{ZnL}{ }^{1}\right]$ $=5.42 \times 10^{-7} \mathrm{M} ; \lambda=428 \mathrm{~nm}$; b) Fluorescence: $\left[\mathrm{ZnL}^{1}\right]=5.33 \times 10^{-7} \mathrm{M} ; \lambda_{\text {exc }}=$ $424 \mathrm{~nm}$.

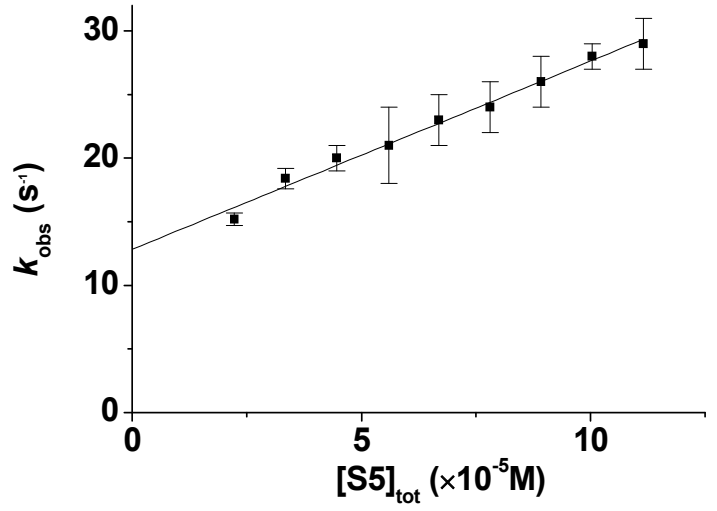

a)

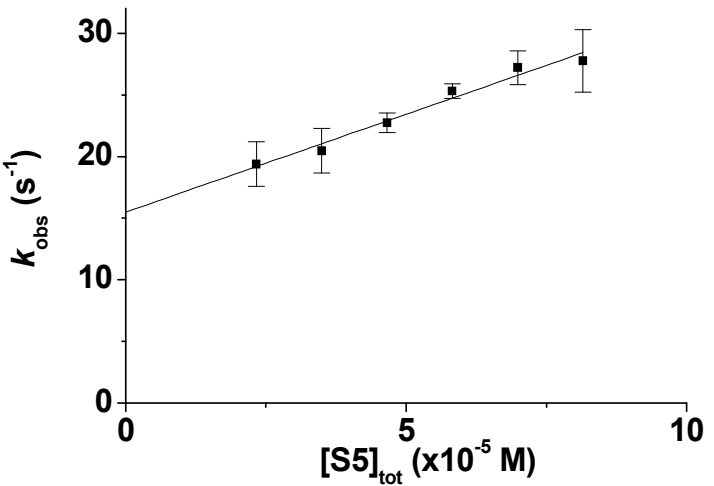

b)

Figure S14. Variation of the pseudo-first order rate constants $\left(k_{\text {obs }}\right)$ for the formation of $\mathrm{ZnL} \boldsymbol{L}^{1}$-S5 complex versus $[\mathbf{S 5}]_{\text {tot. }}$ Solvent: 1,2 -dichloroethane; $T=25.0(2){ }^{\circ} \mathrm{C}$; a) absorption: $\left[\mathrm{Zn} \boldsymbol{L}^{1}\right]=5.42 \times 10^{-7} \mathrm{M} ; \lambda=428 \mathrm{~nm}$; b) fluorescence: $\left[\mathrm{Zn} \boldsymbol{L}^{1}\right]=$ $5.33 \times 10^{-7} \mathrm{M} ; \lambda_{\text {exc }}=424 \mathrm{~nm}$. 


\section{Activation Parameters for the $\mathrm{ZnL}^{1}-\mathrm{S} 4$ Complex}

\begin{tabular}{c|cccccc}
\hline & \multicolumn{7}{|c}{$k_{\mathrm{obs}}\left(\mathrm{s}^{-1}\right)$} \\
$\begin{array}{c}{[\mathbf{S} 4]_{\mathrm{tot}}} \\
\left(\times 10^{-5} \mathrm{M}\right)\end{array}$ & $21.4(2){ }^{\circ} \mathrm{C}$ & $25.4(2){ }^{\circ} \mathrm{C}$ & $28.6(2){ }^{\circ} \mathrm{C}$ & $30.4(2){ }^{\circ} \mathrm{C}$ & $32.7(2){ }^{\circ} \mathrm{C}$ & $37.6(2){ }^{\circ} \mathrm{C}$ \\
\hline 5.08 & $1.3(1)$ & $2.00(4)$ & $2.6(1)$ & $2.9(1)$ & $3.4(2)$ & $4.8(3)$ \\
7.61 & $1.71(8)$ & $2.4(1)$ & $3.06(8)$ & $3.4(1)$ & $4.0(1)$ & $5.58(5)$ \\
10.2 & $1.8(1)$ & $2.6(1)$ & $3.3(1)$ & $3.7(1)$ & $4.3(1)$ & $6.2(2)$ \\
15.2 & $2.3(1)$ & $3.3(1)$ & $4.1(1)$ & $4.6(2)$ & $5.2(2)$ & $7.5(2)$ \\
20.3 & $2.9(2)$ & $3.9(2)$ & $5.0(2)$ & $5.5(2)$ & $6.2(3)$ & $8.9(3)$ \\
25.7 & $3.5(2)$ & $4.8(2)$ & $6.0(3)$ & $6.6(3)$ & $7.6(4)$ & $10.6(5)$ \\
\hline
\end{tabular}

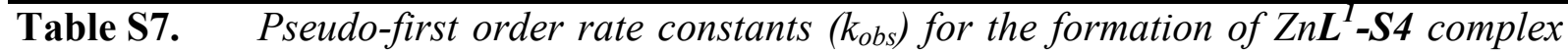
versus $[\mathbf{S} 4]_{\text {tot }}$ measured at different temperatures. Solvent: 1,2-dichloroethane; $\left[Z n \boldsymbol{L}^{1}\right]=1.02 \times 10^{-6} \mathrm{M} ; \lambda=441 \mathrm{~nm}$.

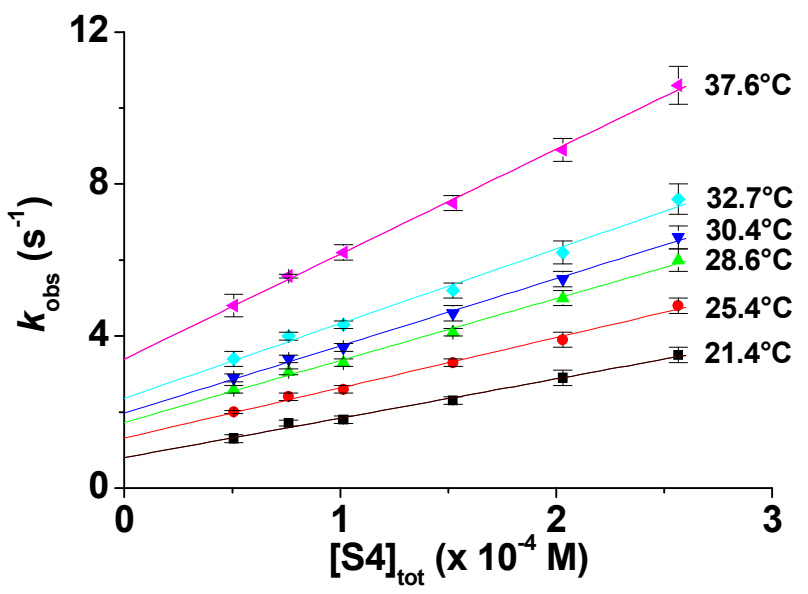

Figure S15. Variation of the pseudo-first order rate constants $\left(k_{o b s}\right)$ for the formation of $\mathrm{Zn} \boldsymbol{L}^{\boldsymbol{1}} \mathbf{- S 4}$ complex versus $[\boldsymbol{S} 4]_{\text {tot }}$ and measured at different temperatures. Solvent: 1,2-dichloroethane; $\left[\mathrm{Zn} \boldsymbol{L}^{1}\right]=1.02 \times 10^{-6} \mathrm{M} ; \lambda=441 \mathrm{~nm}$. 


\begin{tabular}{c|cc}
\hline Temperature $\left({ }^{\circ} \mathrm{C}\right)$ & $k_{f}^{T}\left(\mathrm{M}^{-1} \mathrm{~s}^{-1}\right)$ & $k_{d}^{T}\left(\mathrm{~s}^{-1}\right)$ \\
\hline $21.4(2)$ & $1.04(5) \times 10^{4}$ & $0.80(7)$ \\
$25.4(2)$ & $1.32(5) \times 10^{4}$ & $1.31(8)$ \\
$28.6(2)$ & $1.63(6) \times 10^{4}$ & $1.73(9)$ \\
$30.4(2)$ & $1.77(5) \times 10^{4}$ & $1.97(8)$ \\
$32.7(2)$ & $1.97(5) \times 10^{4}$ & $2.4(2)$ \\
$35.5(2)$ & $2.76(6) \times 10^{4}$ & $3.39(9)$ \\
\hline
\end{tabular}

Table S8. Rate constants for the formation $\left(k_{f}^{T}\right)$ and the dissociation $\left(k_{d}^{T}\right)$ of the selfassembly mechanism of $\boldsymbol{Z n L ^ { 1 } - \boldsymbol { S } 4}$ complex determined at different temperatures. Solvent: 1,2-dichloroethane.

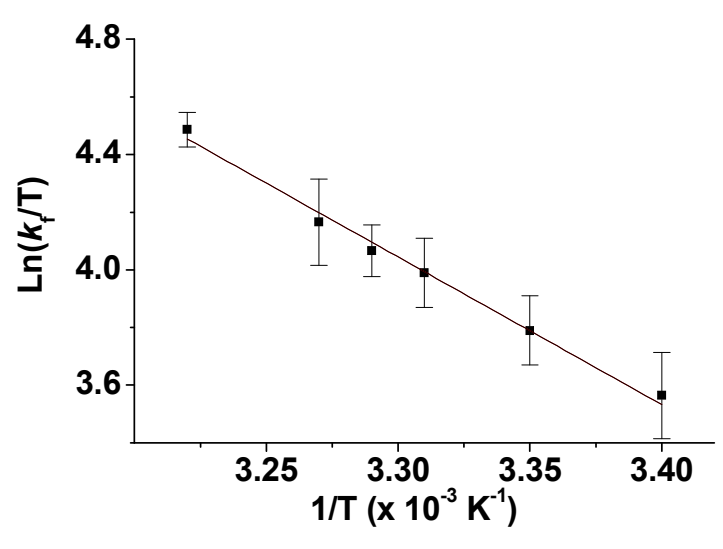

a)

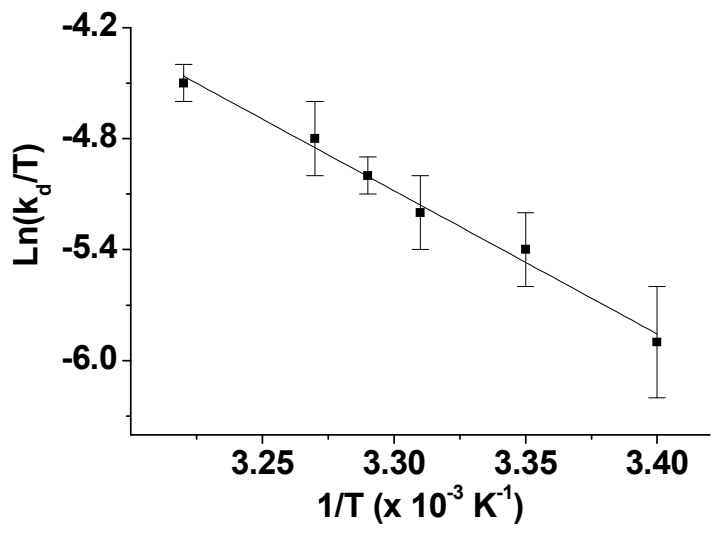

b)

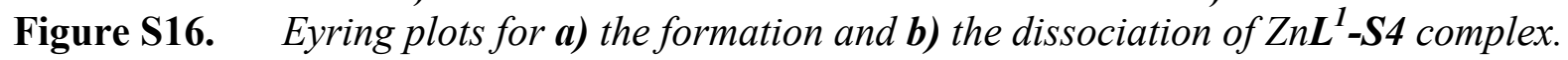
Solvent: 1,2-dichloroethane. 


\section{Spectrophotometric Titrations (UV-vis absorption) of $\mathrm{ZnL}^{2}$ Receptor by Substrates $S$.}

\begin{tabular}{lll}
\hline S1 & {$[\mathbf{S}] /(\mathrm{M})$} & {$\left[\mathbf{Z n L}^{2}\right] /(\mathrm{M})$} \\
\hline S2 & $1.52 \times 10^{-3}$ & $1.01 \times 10^{-6}$ \\
& $2.16 \times 10^{-5}$ & $1.01 \times 10^{-6}$ \\
\end{tabular}

Table S9. Experimental conditions for spectrophotometric titrations (UV-vis absorption) of $Z n \boldsymbol{L}^{2}$ receptor by substrates $\boldsymbol{S 1 - S 5}$. Solvent: 1,2-dichloroethane; $T=25.0(2)$ ${ }^{\circ} \mathrm{C}$.

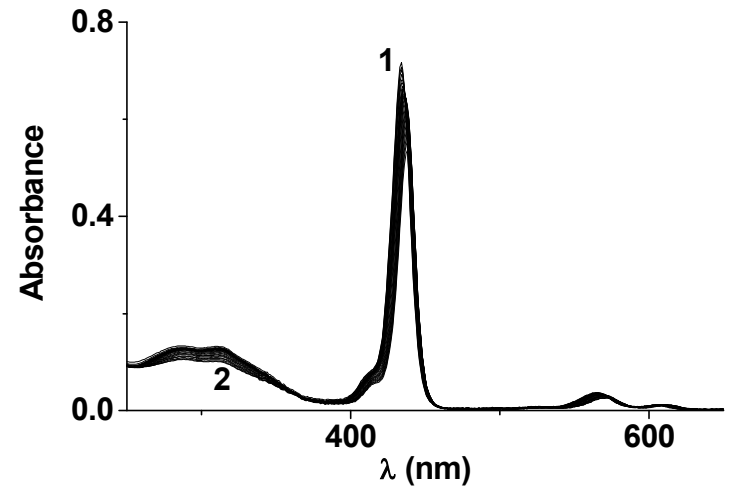

a)

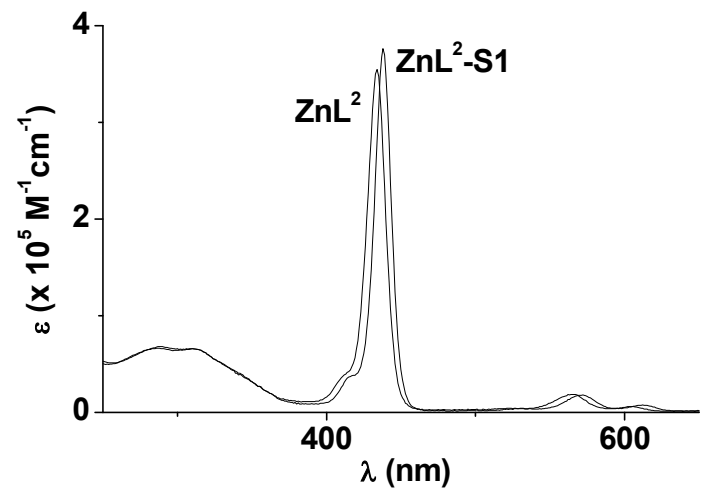

b)

Figure S17. a) UV-visible absorption spectrophotometric titration of $\mathrm{ZnL}^{2}$ receptor versus 1-methyl-1H-imidazole concentrations (noted S1). Solvent: 1,2dichloroethane; $T=25.0(2){ }^{\circ} \mathrm{C} ; \mathrm{l}=2 \mathrm{~cm}$; (1) $\left[\mathrm{Zn} \boldsymbol{L}^{2}\right]_{\text {tot }}=1.01 \times 10^{-6} \mathrm{M}$; (2) $[\boldsymbol{S} 1]_{\text {tot }} /\left[\mathrm{Zn \boldsymbol {L } ^ { 2 }}\right]_{\text {tot }}=2942.5$.

b) Electronic spectra. 


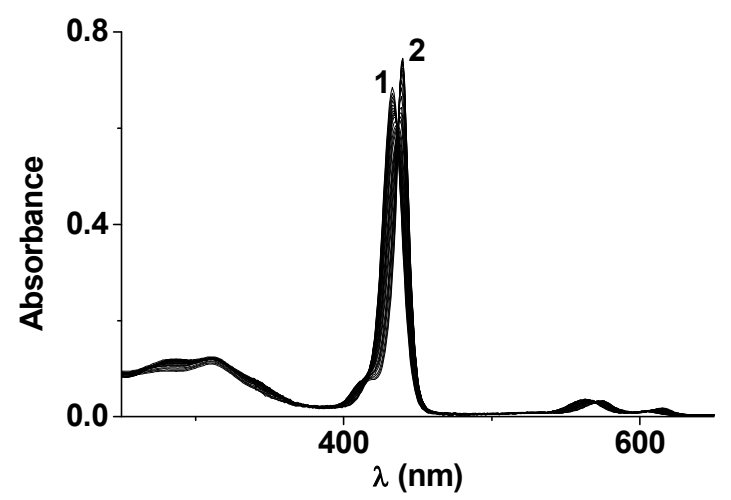

a)

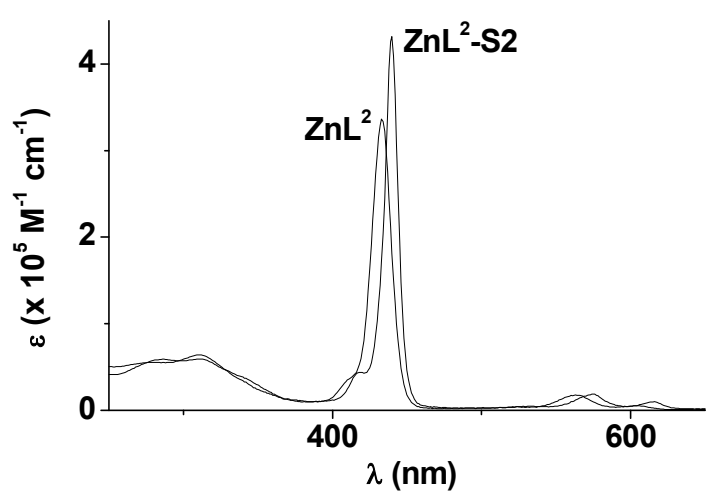

b)

Figure S18. a) UV-visible absorption spectrophotometric titration of $\mathrm{ZnL}^{2}$ receptor versus 1H-imidazole concentrations (noted S2). Solvent: 1,2-dichloroethane; $T=$ $25.0(2){ }^{\circ} \mathrm{C} ; l=2 \mathrm{~cm}$; (1) $\left[\mathrm{Zn} \boldsymbol{L}^{2}\right]_{\text {tot }}=1.01 \times 10^{-6} \mathrm{M}$; (2) $[\mathbf{S} 2]_{\text {tot }} /\left[\mathrm{Zn} \boldsymbol{L}^{2}\right]_{\text {tot }}=$ 3.11 .

b) Electronic spectra.

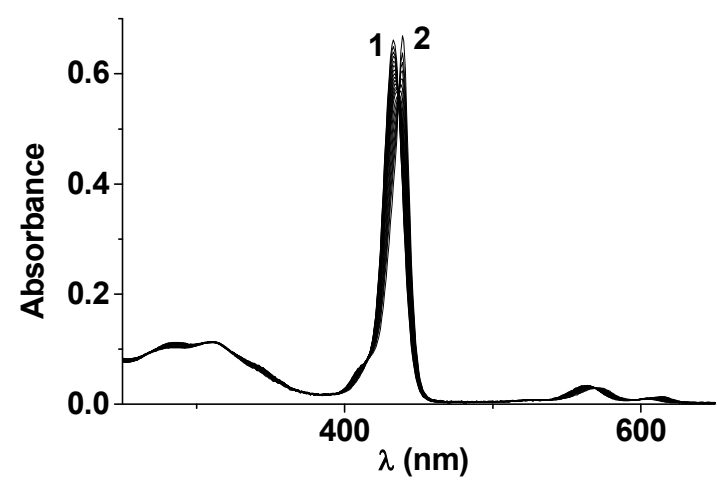

a)

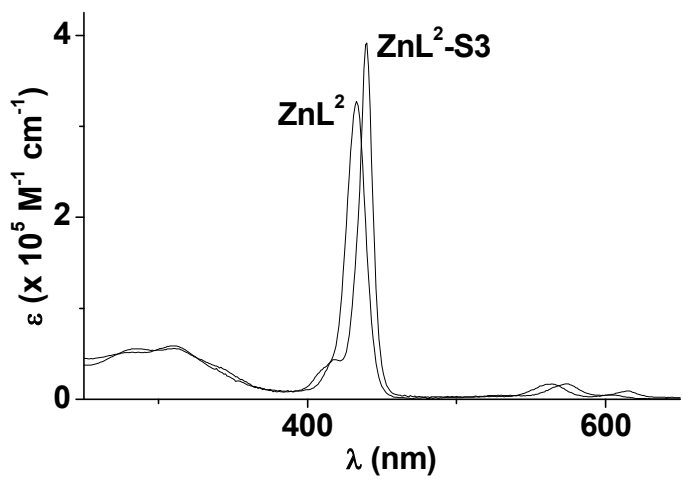

b)

Figure S19. a) UV-visible absorption spectrophotometric titration of $\mathrm{ZnL}^{2}$ receptor versus 2-methyl-1H-imidazole concentrations (noted S3). Solvent: 1,2dichloroethane; $T=25.0(2){ }^{\circ} \mathrm{C} ; \mathrm{l}=2 \mathrm{~cm}$; (1) $\left[\mathrm{Zn} \boldsymbol{L}^{2}\right]_{t o t}=1.01 \times 10^{-6} \mathrm{M}$; (2) $[\mathbf{S 3}]_{\text {tot }} /\left[\mathrm{Zn} \boldsymbol{L}^{2}\right]_{\text {tot }}=0.83$.

b) Electronic spectra. 


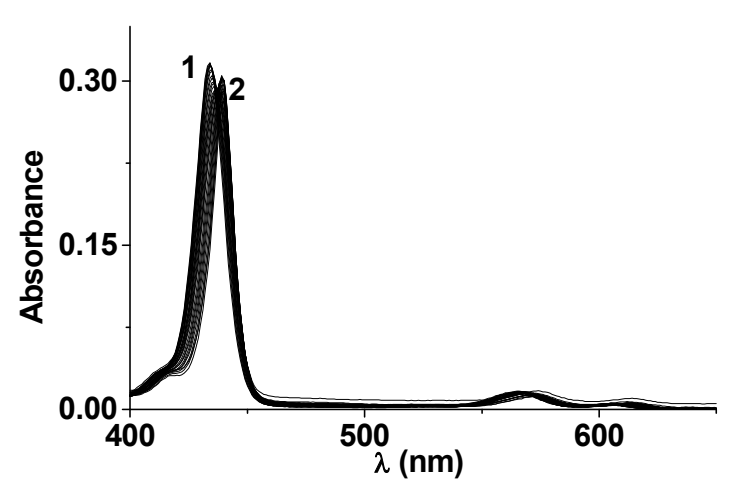

a)

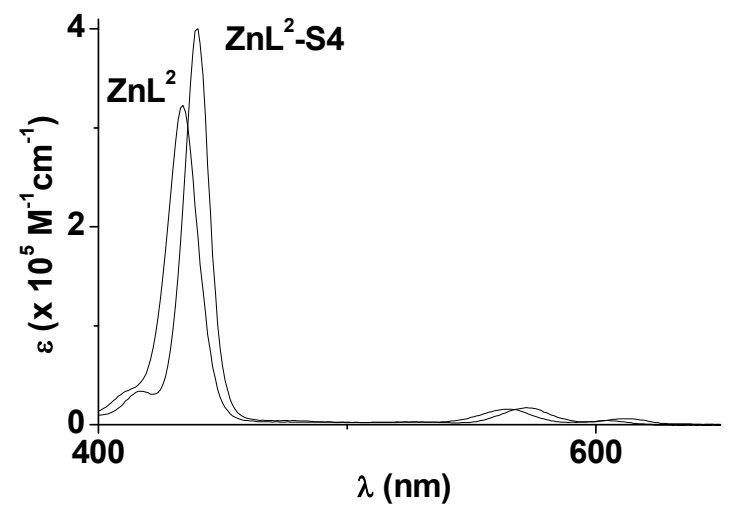

b)

Figure S20. a) UV-visible absorption spectrophotometric titration of $Z n \mathbf{L} 2^{1}$ receptor versus 2-phenyl-1H-imidazole concentrations (noted $\boldsymbol{S 4}$. Solvent: 1,2-dichloroethane; $T=25.0(2){ }^{\circ} \mathrm{C} ; l=1 \mathrm{~cm}$; (1) $\left[\mathrm{Zn} \boldsymbol{L}^{2}\right]_{\text {tot }}=1.01 \times 10^{-6} \mathrm{M}$; (2) $[\mathbf{S} 4]_{\text {tot }} /\left[\mathrm{Zn} \boldsymbol{L}^{2}\right]_{\text {tot }}=$ 23360.39 .

b) Electronic spectra.

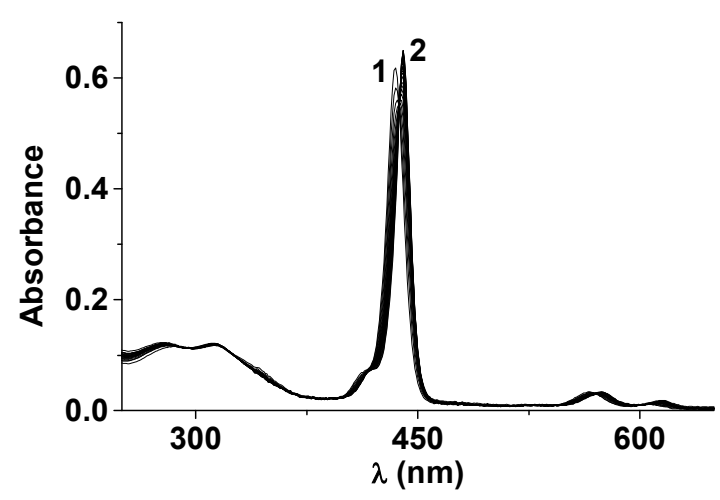

a)

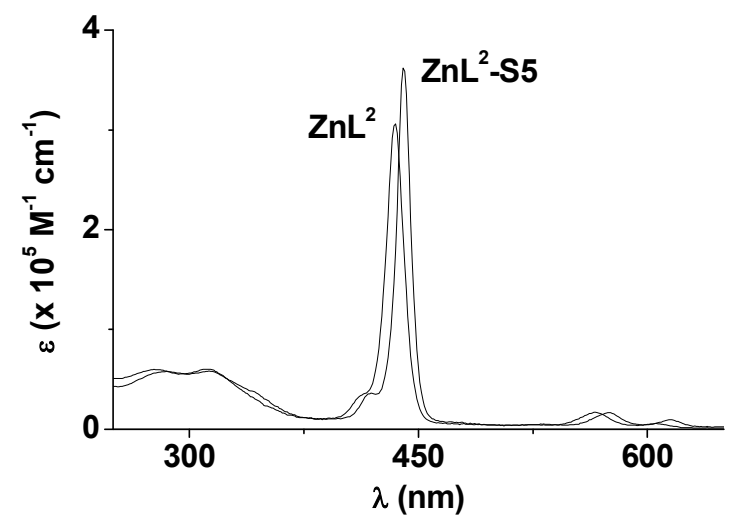

b)

Figure S21. a) UV-visible absorption spectrophotometric titration of $\mathrm{ZnL}^{2}$ receptor versus $N$-acetyl-(L)-histidine methyl ester concentrations (noted S5). Solvent: 1,2dichloroethane; $T=25.0(2){ }^{\circ} \mathrm{C} ; \mathrm{l}=2 \mathrm{~cm} ;$ (1) $\left[\mathrm{Zn \mathbf {L } ^ { 2 }}\right]_{\text {tot }}=1.01 \times 10^{-6} \mathrm{M}$; (2) $[\mathbf{S} 5]_{\text {tot }} /\left[\mathrm{ZnL}^{2}\right]_{\text {tot }}=89.63$.

b) Electronic spectra. 


\section{Luminescence Titrations of $\mathrm{ZnL}^{2}$ Receptor by Substrates S.}

\begin{tabular}{lccc}
\hline S1 & {$[\mathbf{S}] / \mathrm{M}$} & {$\left[\mathbf{Z n L}^{2}\right] / \mathrm{M}$} & $\lambda_{\text {exc }} / \mathrm{nm}$ \\
\hline S3 & $7.52 \times 10^{-3}$ & $5.07 \times 10^{-7}$ & 436 \\
& & & $4.05 \times 10^{-6}$ \\
S5 & $5.41 \times 10^{-6}$ & $5.07 \times 10^{-7}$ & 436 \\
\end{tabular}

Table S10. Experimental conditions for luminescence titrations of $\mathrm{ZnL}^{2}$ receptor by substrates S1-S5. Solvent: 1,2-dichloroethane; $T=25.0(2){ }^{\circ} \mathrm{C}$; the slit width was set at $15 \mathrm{~nm}$ for both the excitation and the emission.

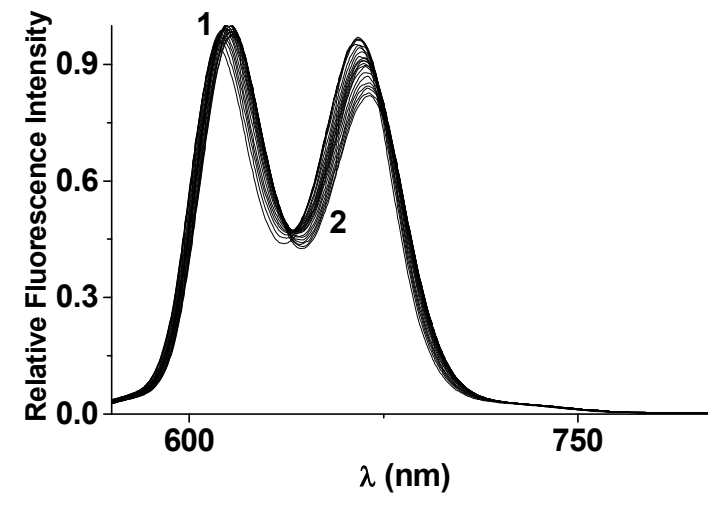

a)

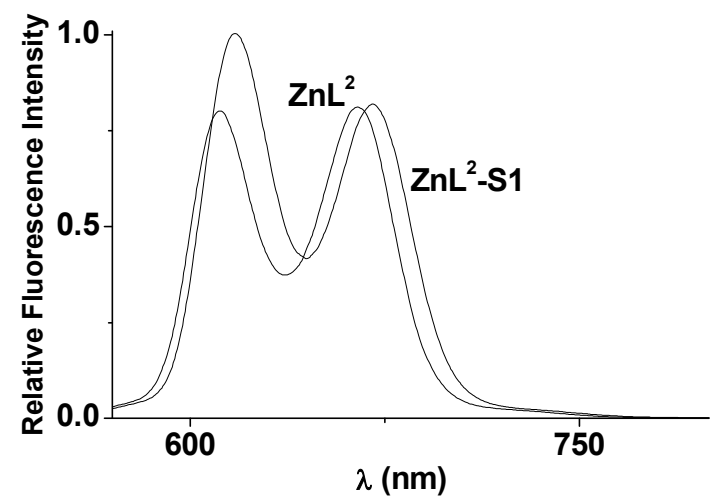

b)

Figure S22. a) Spectrofluorimetric titration of $\mathrm{Zn \mathbf {L } ^ { 2 }}$ receptor versus 1-methyl-1H-imidazole concentrations (noted S1). Solvent: 1,2-dichloroethane; $T=25.0(2){ }^{\circ} \mathrm{C} ; \lambda_{\text {exc }}=$ $436 \mathrm{~nm}$; (1) $\left[\mathrm{Zn} \boldsymbol{L}^{2}\right]_{\text {tot }}=5.07 \times 10^{-7} \mathrm{M}$; (2) $[\mathbf{S} 1]_{\text {tot }} /\left[\mathrm{Zn} \boldsymbol{L}^{2}\right]_{\text {tot }}=1929.23$.

b) Relative recalculated luminescence spectra. 


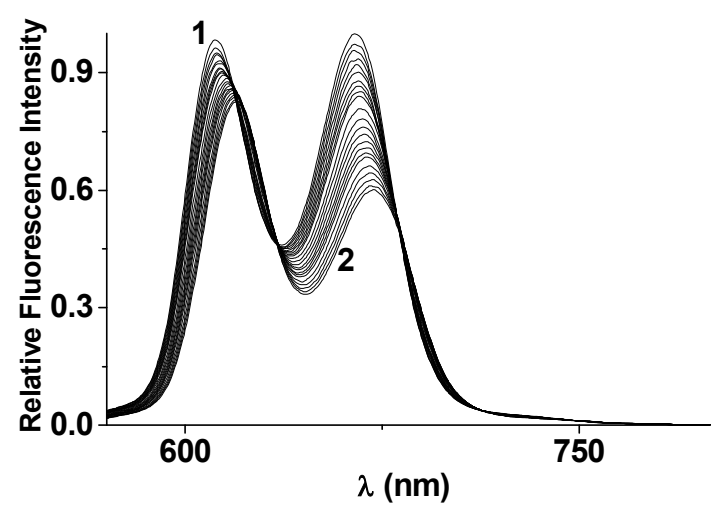

a)

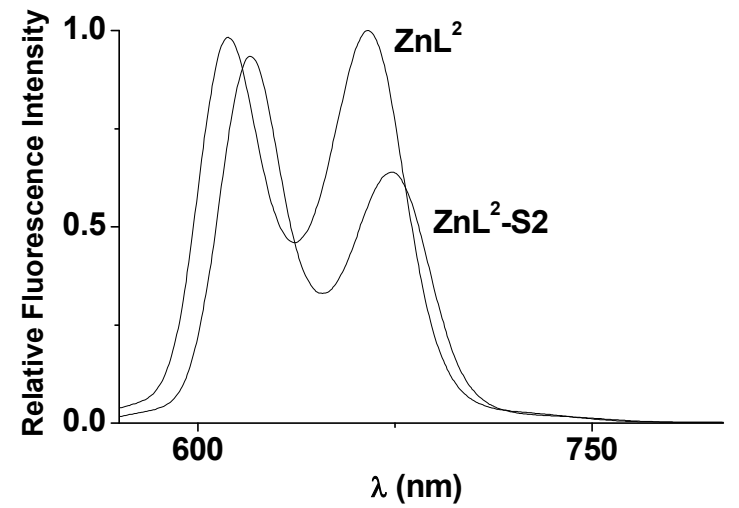

b)

Figure S23. a) Spectrofluorimetric titration of $\mathrm{ZnL}^{2}$ receptor versus 1 H-imidazole concentrations (noted $\boldsymbol{S} 2$ ). Solvent: 1,2-dichloroethane; $T=25.0(2){ }^{\circ} \mathrm{C} ; \lambda_{\text {exc }}=$ $436 \mathrm{~nm}$; (1) $\left[\mathrm{Zn} \boldsymbol{L}^{2}\right]_{\text {tot }}=5.07 \times 10^{-7} \mathrm{M}$; (2) $[\mathbf{S 1}]_{\text {tot }} /\left[\mathrm{Zn} \boldsymbol{L}^{2}\right]_{\text {tot }}=2.01$.

b) Relative recalculated luminescence spectra.

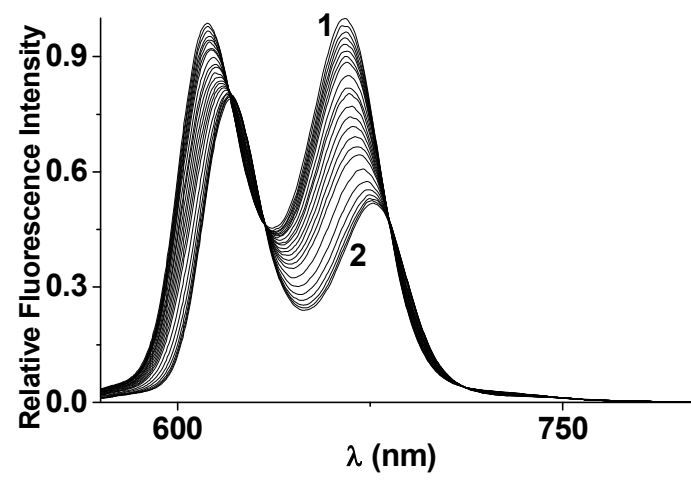

a)

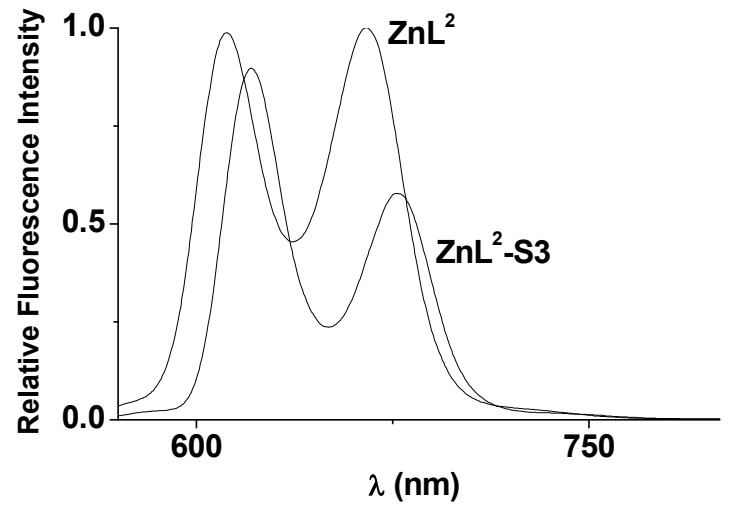

b)

Figure S24. a) Spectrofluorimetric titration of $\mathrm{ZnL}^{2}$ receptor versus 2-methyl-1H-imidazole concentrations (noted S3). Solvent: 1,2-dichloroethane; $T=25.0(2){ }^{\circ} \mathrm{C} ; \lambda_{\text {exc }}=$ $436 \mathrm{~nm}$; (1) $\left[\mathrm{Zn} \boldsymbol{L}^{2}\right]_{\text {tot }}=5.07 \times 10^{-7} \mathrm{M}$; (2) $[\mathbf{S} 3]_{\text {tot }} /\left[\mathrm{Zn} \boldsymbol{L}^{2}\right]_{\text {tot }}=1.39$.

b) Relative recalculated luminescence spectra. 


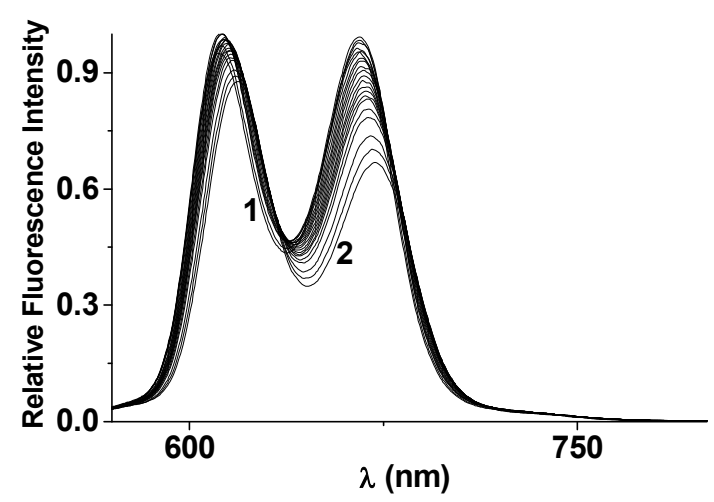

a)

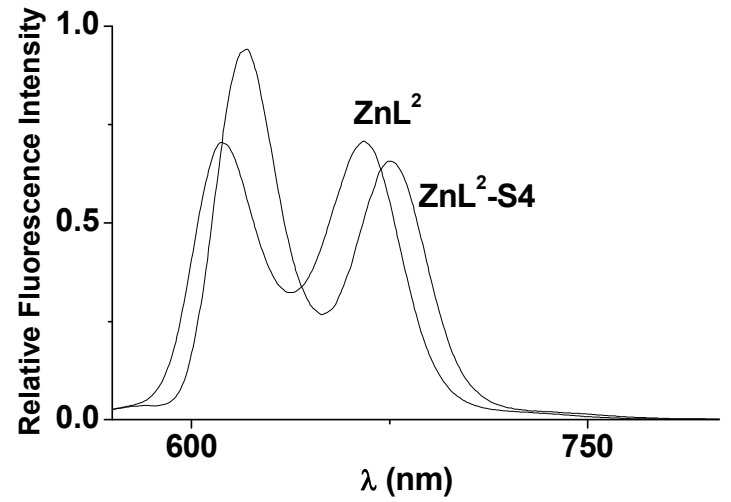

b)

Figure S25. a) Spectrofluorimetric titration of $\mathrm{ZnL}^{2}$ receptor versus 2-phenyl-1H-imidazole concentrations (noted S4). Solvent: 1,2-dichloroethane; $T=25.0(2){ }^{\circ} \mathrm{C} ; \lambda_{\text {exc }}=$ $436 \mathrm{~nm}$; (1) $\left[\mathrm{Zn} \boldsymbol{L}^{2}\right]_{\text {tot }}=5.07 \times 10^{-7} \mathrm{M}$; (2) $[\mathbf{S} 4]_{\text {tot }} /\left[\mathrm{Zn \boldsymbol {L } ^ { 2 }}\right]_{\text {tot }}=15526.6$.

b) Relative recalculated luminescence spectra.

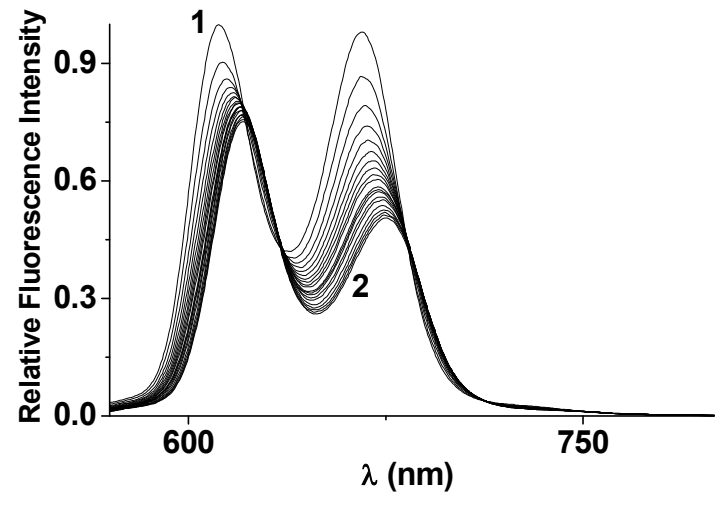

a)

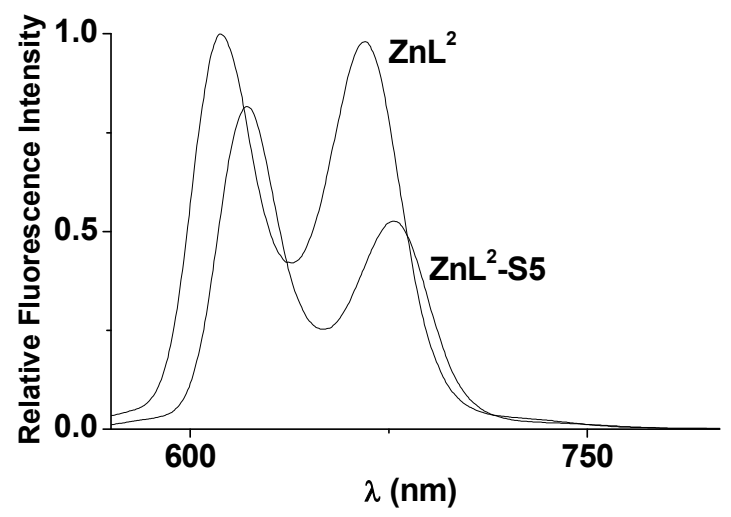

b)

Figure S26. a) Spectrofluorimetric titration of $\mathrm{ZnL}^{2}$ receptor versus $N$-acetyl-(L)-histidine methyl ester concentrations (noted S5). Solvent: 1,2-dichloroethane; $T=$ $25.0(2){ }^{\circ} \mathrm{C}$; $\lambda_{\text {exc }}=437 \mathrm{~nm}$; (1) $\left[\mathrm{Zn} \boldsymbol{L}^{2}\right]_{\text {tot }}=5.07 \times 10^{-7} \mathrm{M}$; (2) $[\mathbf{S 5}]_{\text {tot }} /\left[\mathrm{Zn} \boldsymbol{L}^{2}\right]_{\text {tot }}$ $=106.5$.

b) Relative recalculated spectra. 
Kinetic Studies (Receptor $\mathrm{ZnL}^{2}$ and Substrate S3)<smiles>Cc1ncc[nH]1</smiles>

\begin{tabular}{cccc}
\hline $\begin{array}{c}{[\mathbf{S 3}]_{\mathrm{tot}}} \\
\left(\times 10^{-5} \mathrm{M}\right)\end{array}$ & $k_{\mathrm{obs}} \pm 3 \sigma\left(\mathrm{s}^{-1}\right)$ & $\begin{array}{c}{[\mathbf{S 3}]_{\mathrm{tot}}} \\
\left(\times 10^{-5} \mathrm{M}\right)\end{array}$ & $k_{\mathrm{obs}} \pm 3 \sigma\left(\mathrm{s}^{-1}\right)$ \\
\hline \multicolumn{2}{c}{ Absorption } & \multicolumn{2}{c}{ Fluorescence } \\
1.25 & $26(3)$ & 0.52 & $28(5)$ \\
3.37 & $73.5(9)$ & 0.78 & $30(3)$ \\
3.85 & $93(3)$ & 1.04 & $50(8)$ \\
4.43 & $122(7)$ & 1.30 & $63(10)$ \\
5.00 & $123(11)$ & 1.56 & $64(3)$ \\
5.77 & $144(8)$ & 2.08 & $95(8)$
\end{tabular}

Table S11. Variation of the pseudo-first order rate constants versus $[\mathbf{S} 3]_{\text {tot }}$ for $\mathrm{Zn \mathbf {L } ^ { 2 } - \mathbf { S } 3}$ complex. Solvent: 1,2-dichloroethane; $T=25.0(2){ }^{\circ} \mathrm{C}$; a) Absorption: $\left[\mathrm{ZnL}^{2}\right]$ $=1.34 \times 10^{-6} \mathrm{M} ; \lambda=440 \mathrm{~nm}$; b) Fluorescence: $\left[\mathrm{ZnL}^{2}\right]=2.67 \times 10^{-7} \mathrm{M} ; \lambda_{\text {exc }}=$ $436 \mathrm{~nm}$.

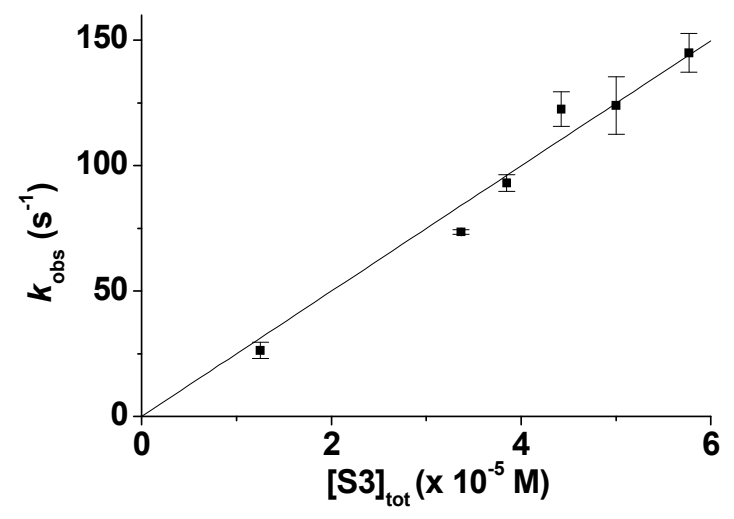

a)

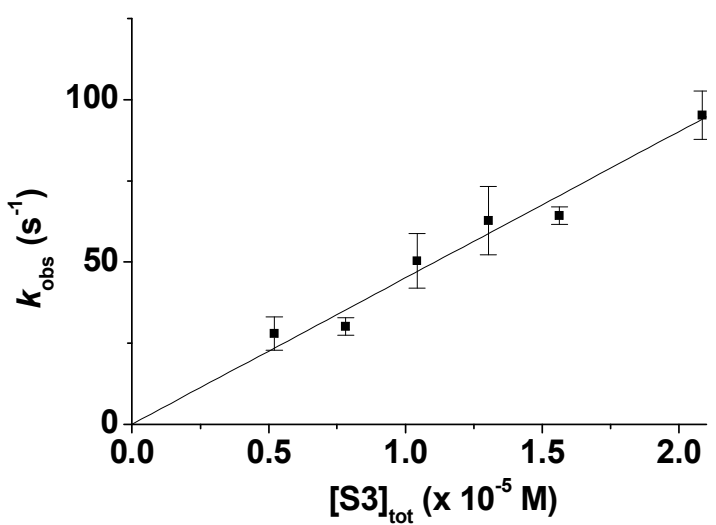

b)

Figure S27. Variation of the pseudo-first order rate constants ( $\left.k_{o b s}\right)$ for the formation of $\mathrm{Zn} \boldsymbol{L}^{2}-\mathbf{S} 3$ complex versus $[\boldsymbol{S 3}]_{\text {tot }}$ Solvent: 1,2-dichloroethane; $T=25.0(2){ }^{\circ} \mathrm{C}$; a) absorption: $\left[\mathrm{ZnL}^{2}\right]=1.34 \times 10^{-6} \mathrm{M} ; \lambda=440 \mathrm{~nm}$; b) Fluorescence: $\left[\mathrm{Zn} \boldsymbol{L}^{2}\right]$ $=2.67 \times 10^{-7} \mathrm{M} ; \lambda_{\text {exc }}=436 \mathrm{~nm}$. 


\section{Kinetic studies (receptor $\mathrm{ZnL}^{2}$ and substrate S4)}

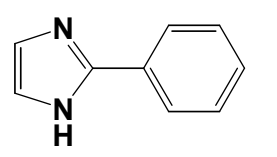

\begin{tabular}{cccc}
\hline $\begin{array}{c}{[\mathbf{S 4}]_{\mathrm{tot}}} \\
\left(\times 10^{-3} \mathrm{M}\right)\end{array}$ & $k_{\mathrm{obs}} \pm 3 \sigma\left(\mathrm{s}^{-1}\right)$ & $\begin{array}{c}{[\mathbf{S} 4]_{\mathrm{tot}}} \\
\left(\times 10^{-3} \mathrm{M}\right)\end{array}$ & $k_{\mathrm{obs}} \pm 3 \sigma\left(\mathrm{s}^{-1}\right)$ \\
\hline \multicolumn{2}{c}{ Absorption } & \multicolumn{2}{c}{ Fluorescence } \\
1.0 & $4.9(4)$ & 2.0 & $5.2(9)$ \\
2.0 & $5.6(3)$ & 3.0 & $5.5(6)$ \\
3.0 & $6.1(3)$ & 4.0 & $6.0(9)$ \\
4.0 & $6.5(6)$ & 5.0 & $6.4(9)$ \\
5.0 & $6.9(4)$ & 6.0 & $7.3(9)$ \\
6.0 & $7.7(8)$ & 7.0 & $7.4(6)$ \\
7.0 & $7.9(3)$ & &
\end{tabular}

Table S12. Variation of the pseudo-first order rate constants versus $[\mathbf{S} 4]_{\text {tot }}$ for $\mathrm{Zn \mathbf {L } ^ { 2 } - \mathbf { S } 4}$ complex. Solvent: 1,2-dichloroethane; $T=25.0(2){ }^{\circ} \mathrm{C}$; a) absorption: $\left[\mathrm{Zn} \boldsymbol{L}^{2}\right]=$ $5 \times 10^{-6} \mathrm{M} ; \lambda=441 \mathrm{~nm}$; b) fluorescence: $\left[\mathrm{Zn} \boldsymbol{L}^{2}\right]=5 \times 10^{-6} \mathrm{M} ; \lambda_{\text {exc }}=567 \mathrm{~nm}$.

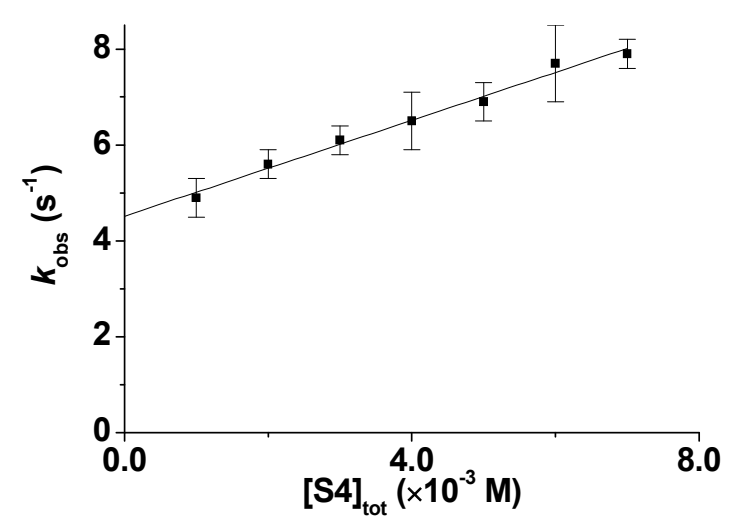

a)

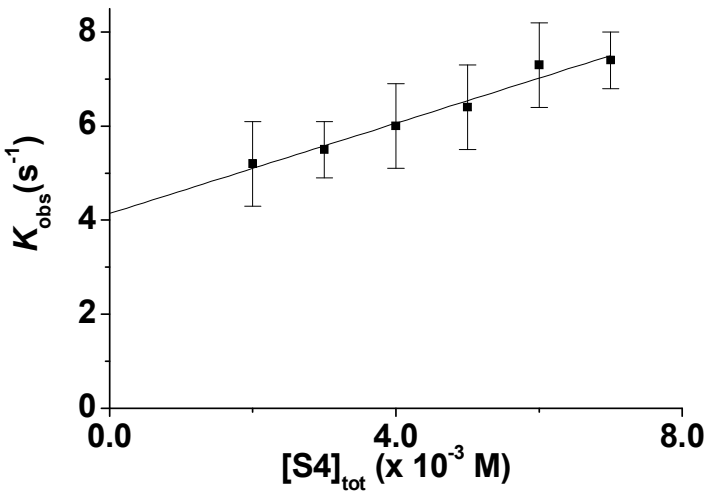

b)

Figure S28. Variation of the pseudo-first order rate constants $\left(k_{\text {obs }}\right)$ for the formation of $\mathrm{Zn} \boldsymbol{L}^{2}-\boldsymbol{S} 4$ complex versus [S4] tot. Solvent: 1,2-dichloroethane; $T=25.0(2){ }^{\circ} \mathrm{C}$; a) absorption: $\left[\mathrm{Zn} \boldsymbol{L}^{2}\right]=5 \times 10^{-6} \mathrm{M} ; \lambda=441 \mathrm{~nm}$; b) fluorescence: $\left[\mathrm{Zn} \boldsymbol{L}^{2}\right]=5$ $\times 10^{-6} \mathrm{M} ; \lambda_{\text {exc }}=567 \mathrm{~nm}$. 


\section{Kinetic studies (receptor $\mathrm{ZnL}^{2}$ and substrate S5)}
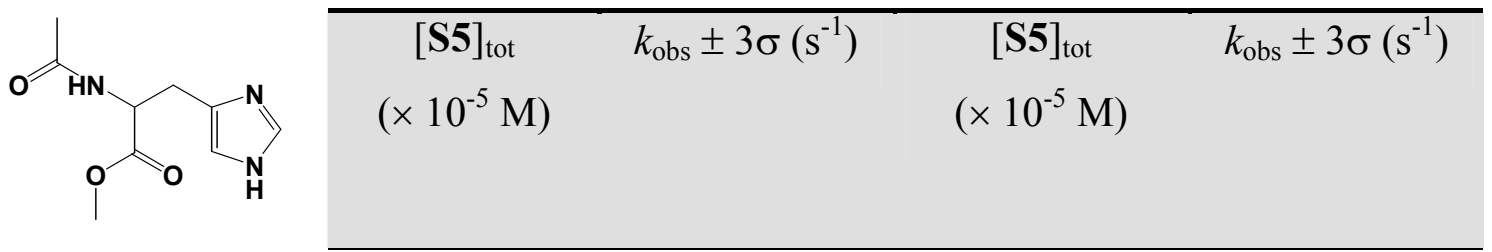

\begin{tabular}{llll}
\hline \multicolumn{2}{c}{ Absorption } & \multicolumn{2}{c}{ Fluorescence } \\
0.50 & $0.60(3)$ & 1.04 & $0.6(3)$ \\
1.00 & $0.82(6)$ & 2.08 & $0.9(5)$ \\
1.94 & $1.16(6)$ & 3.13 & $1.2(2)$ \\
2.99 & $1.3(1)$ & 4.17 & $1.7(6)$ \\
3.98 & $1.6(1)$ & 5.21 & $1.7(4)$ \\
4.98 & $1.6(1)$ & 6.25 & $1.7(1)$ \\
5.98 & $1.7(2)$ & & \\
\hline
\end{tabular}

Table S13. Variation of the pseudo-first order rate constants versus $[\mathbf{S 5}]_{\text {tot }}$ for $\mathrm{Zn \mathbf {L } ^ { 2 } - \mathrm { S } 5}$ complex. Solvent: 1,2-dichloroethane; $T=25.0(2){ }^{\circ} \mathrm{C}$; a) absorption: $\left[\mathrm{Zn} \boldsymbol{L}^{2}\right]=$ $5.09 \times 10^{-6} \mathrm{M} ; \lambda=440 \mathrm{~nm}$; b) fluorescence: $\left[\mathrm{ZnL}^{2}\right]=5.05 \times 10^{-7} \mathrm{M} ; \lambda_{\text {exc }}=437$ nm.

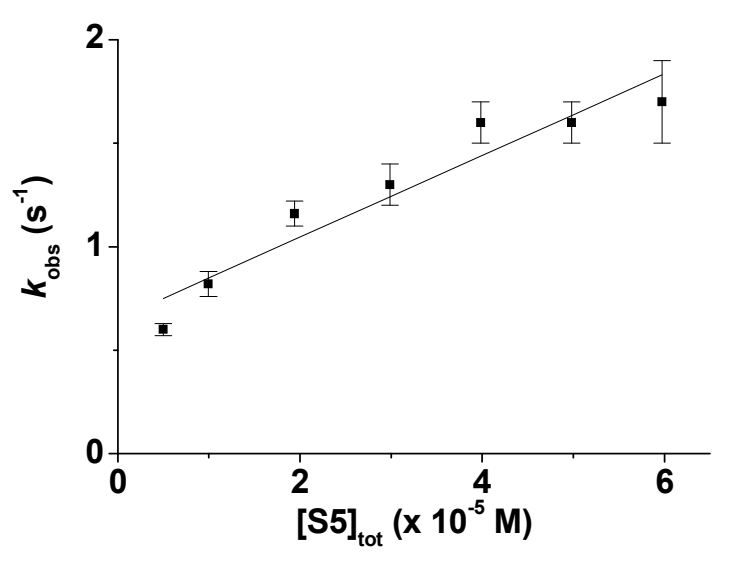

a)

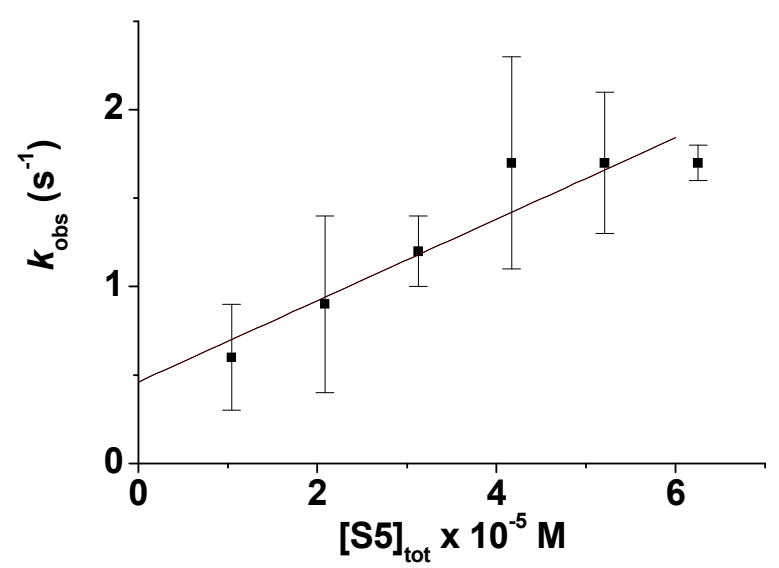

b)

Figure S29. Variation of the pseudo-first order rate constants $\left(k_{o b s}\right)$ for the formation of

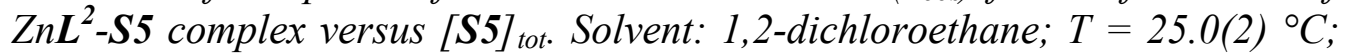
a) absorption: $\left[\mathrm{Zn} \boldsymbol{L}^{2}\right]=5.09 \times 10^{-6} \mathrm{M} ; \lambda=440 \mathrm{~nm}$; b) fluorescence: $\left[\mathrm{Zn} \boldsymbol{L}^{2}\right]=$ $5.05 \times 10^{-6} \mathrm{M} ; \lambda_{\text {exc }}=437 \mathrm{~nm}$. 


\section{Activation parameters for the $\mathrm{ZnL}^{2}-\mathrm{S} 4$ complex}

\begin{tabular}{c|cccccc}
\hline & \multicolumn{7}{|c}{$k_{\mathrm{obs}}\left(\mathrm{s}^{-1}\right)$} \\
{$[\mathbf{S 4}]_{\text {tot }}$} & $21.1(2){ }^{\circ} \mathrm{C}$ & $24.8(2){ }^{\circ} \mathrm{C}$ & $27.1(2){ }^{\circ} \mathrm{C}$ & $30.2(2){ }^{\circ} \mathrm{C}$ & $33.4(2){ }^{\circ} \mathrm{C}$ & $36.0(2){ }^{\circ} \mathrm{C}$ \\
\hline 0.001 & $2.9(4)$ & $4.1(3)$ & $5.3(7)$ & $7(1)$ & $10(1)$ & $12(2)$ \\
0.002 & $3.1(1)$ & $4.7(5)$ & $6.0(5)$ & $8(1)$ & $11.0(6)$ & $13.9(2)$ \\
0.003 & $3.5(2)$ & $5.0(2)$ & $6.5(3)$ & $8.4(5)$ & $12(2)$ & $14.9(2)$ \\
0.004 & $3.6(2)$ & $5.4(5)$ & $6.8(3)$ & $8.9(5)$ & $12.8(6)$ & $15(1)$ \\
0.005 & $3.8(1)$ & $5.4(5)$ & $6.8(7)$ & $9.2(4)$ & $13.1(5)$ & $16(1)$ \\
0.006 & $4.2(3)$ & $6.5(5)$ & $8.0(9)$ & $10.9(9)$ & $14.7(2)$ & $18.5(7)$ \\
0.007 & $4.3(3)$ & $6.6(3)$ & $8.1(3)$ & $11(1)$ & $15.3(7)$ & $19(2)$ \\
\hline
\end{tabular}

Table S14. Pseudo-first order rate constants ( $k_{o b s}$ ) for the formation of $\mathrm{ZnL}^{2}-\mathbf{S} 4$ complex versus [S4] tot measured at different temperatures. Solvent: 1,2-dichloroethane; $l=0.2 \mathrm{~cm} ;\left[\mathrm{ZnL}^{2}\right]=5.23 \times 10^{-6} \mathrm{M} ; \lambda=441 \mathrm{~nm}$.

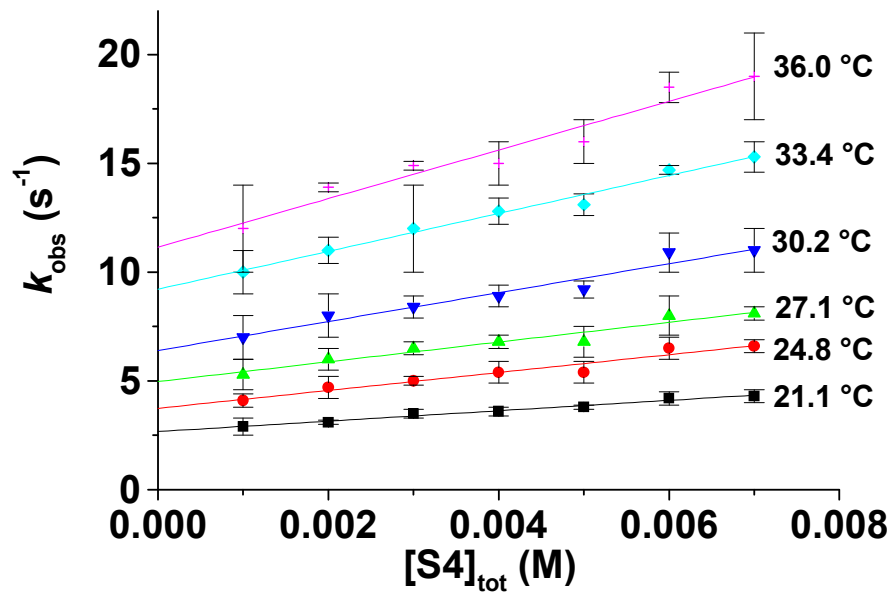

Figure S30. Variation of the pseudo-first order rate constants $\left(k_{\text {obs }}\right)$ for the formation of $Z n \boldsymbol{L}^{2}-\mathbf{S} 4$ complex versus $[\mathbf{S} 4]_{\text {tot }}$ and measured at different temperatures. Solvent: 1,2-dichloroethane; $\left[\mathrm{ZnL}^{2}\right]=5.23 \times 10^{-6} \mathrm{M} ; \lambda=441 \mathrm{~nm}$. 


\begin{tabular}{c|cc}
\hline Temperature $\left({ }^{\circ} \mathrm{C}\right)$ & $k_{f}^{T}\left(\mathrm{M}^{-1} \mathrm{~s}^{-1}\right)$ & $k_{d}{ }^{T}\left(\mathrm{~s}^{-1}\right)$ \\
\hline $21.1(2)$ & $2.4(4) \times 10^{2}$ & $2.7(2)$ \\
$24.8(2)$ & $4(1) \times 10^{2}$ & $3.7(6)$ \\
$27.1(2)$ & $5(1) \times 10^{2}$ & $5.0(7)$ \\
$30.2(2)$ & $7(2) \times 10^{2}$ & $6.4(9)$ \\
$33.4(2)$ & $9(1) \times 10^{2}$ & $9.2(7)$ \\
$36.0(2)$ & $1.1(3) \times 10^{3}$ & $11(2)$ \\
\hline
\end{tabular}

Table S15. Rate constants for the formation $\left(k_{f}^{T}\right)$ and the dissociation $\left(k_{d}^{T}\right)$ for the selfassembly mechanism of $\mathrm{ZnL}^{2} \mathbf{- S} \mathbf{4}$ complex determined at different temperatures. Solvent: 1,2-dichloroethane.

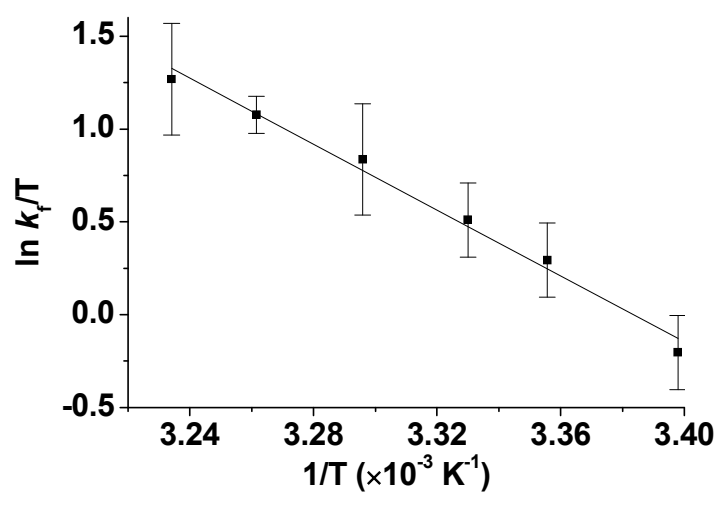

a)

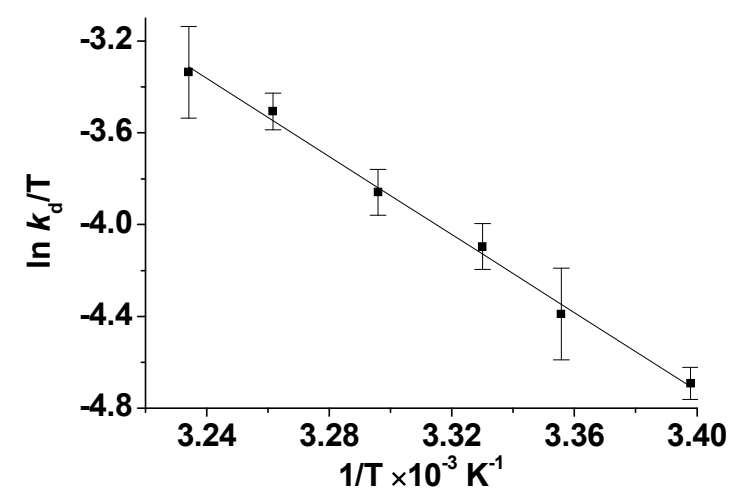

b)

Figure S31. Eyring plots for $\boldsymbol{a})$ the formation and $\boldsymbol{b})$ the dissociation of $\mathrm{ZnL}^{\mathbf{2}} \mathbf{- S} \mathbf{4}$ complex. Solvent: 1,2-dichloroethane. 


\section{Zn(II)-Porphen Receptor: Histidine Complexation}
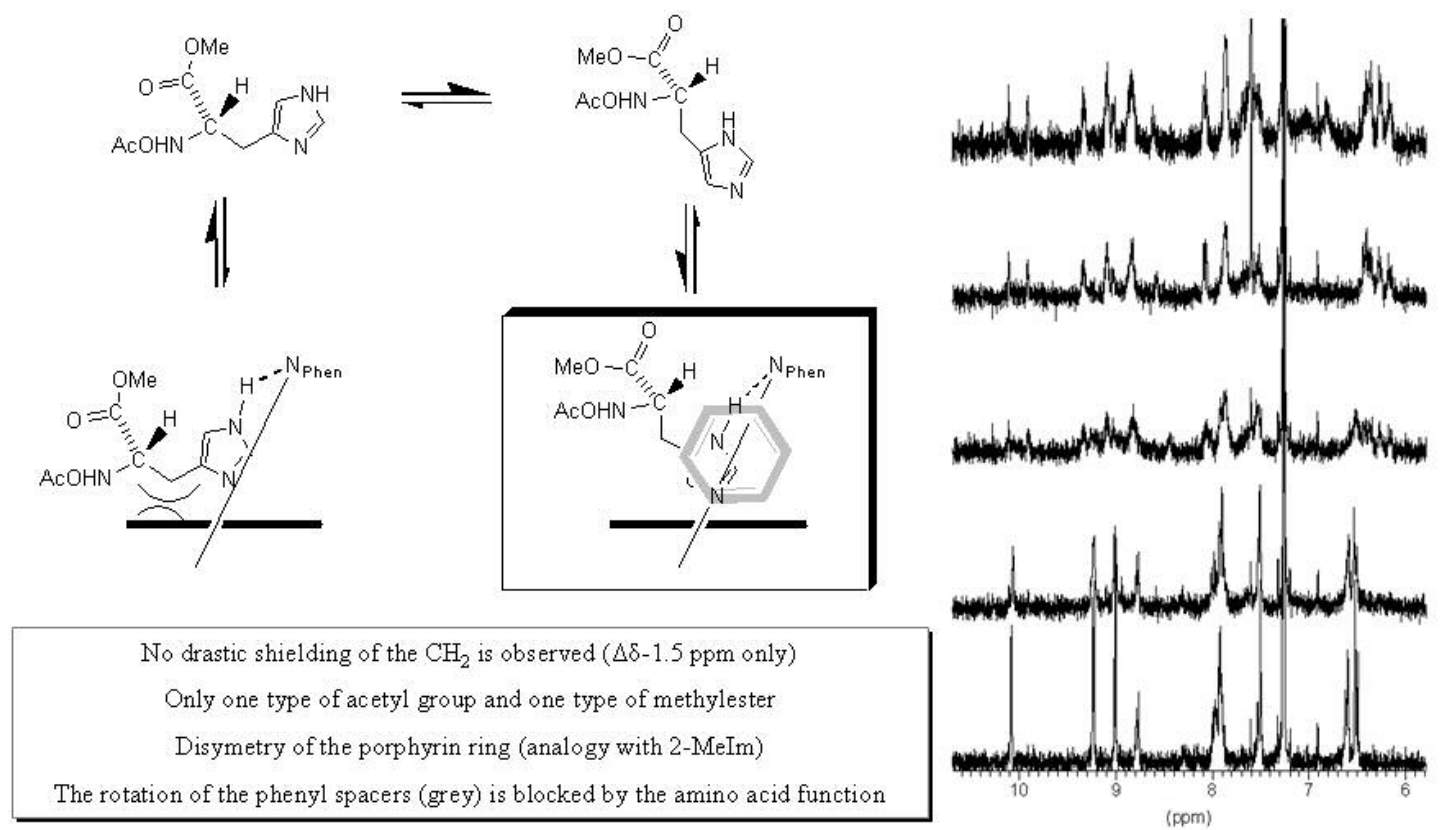

Figure S32. 1H NMR (300 MHz) evolution upon distal binding of $3 H$-tautomer of substrate S5 by zinc(II) porphyrin $\mathrm{ZnL}^{1}$. Solvent: $\mathrm{CDCl}_{3} ; T=298 \mathrm{~K}$. 